\title{
Global molecular gas properties of Seyfert galaxies ${ }^{\star}$
}

\section{Analysis of the results}

\author{
S. J. Curran ${ }^{1,2,3}$, A. G. Polatidis ${ }^{1}$, S. Aalto ${ }^{1}$, and R. S. Booth ${ }^{1}$ \\ 1 Onsala Space Observatory, Chalmers University of Technology, 43992 Onsala, Sweden \\ 2 European Southern Observatory, Casilla 19001, Santiago 19, Chile \\ 3 School of Physics, University of New South Wales, Sydney NSW 2052, Australia \\ Received 3 April 2001 / Accepted 24 April 2001
}

\begin{abstract}
We use the data published in Paper I and Curran et al. (2000) to determine the global molecular gas luminosities and distributions in a sample of 22 Seyfert galaxies. From this we find definite differences in the $\mathrm{CO}$ to HCN luminosity ratios between the near-by and distant galaxies of the sample. This is perhaps due to a selection effect where we only observe the brightest of the distant sources. With regard to distributions, we find in the near-by (mapped) sample that the CO is usually much wider distributed than the central telescope beam and that the $\mathrm{HCN}$ is considerably more extended beyond the $\sim 1 \mathrm{kpc}$ often cited in the literature. In fact this molecule has been detected as far as $\approx 5 \mathrm{kpc}$ from the centre of NGC 1365 . We may also have detected a bar in HCN in NGC 5033. Also, using the data to complement the results of Curran (2000a), we find $\frac{L_{\mathrm{CO}}}{L_{\mathrm{FIR}}}(\mathrm{Sy} 2) \approx \frac{L_{\mathrm{CO}}}{L_{\mathrm{FIR}}}(\mathrm{Sy} 1)$ i.e. no difference in the molecular gas luminosities between the two main Seyfert classes. In fact we consider it more meaningful to discuss the differences between the near-by and distant sample (irrespective of Seyfert type), although both of these samples may show evidence that much of the far infrared luminosity could arise from an active galactic nucleus as opposed to being predominantly due to vigorous star formation. We do believe, however, that improved statistics would be of little value in distinguishing between these two scenarios and that future high resolution observations are the key to resolving this issue.
\end{abstract}

Key words. galaxies: Seyfert - galaxies: starburst - galaxies: abundances - galaxies: ISM

\section{Introduction}

Activity in galaxies spans from centrally located active galactic nuclei (AGN) to more extended but less powerful starbursts, although in some extreme ultra-luminous infrared galaxies (ULIRGs) the star-powered luminosity may rival that of a compact AGN. Both phenomena appear to be associated with significant amounts of circumnuclear gas and high resolution observations reveal that the HCN, which is much more centralised than the CO, tends to trace gas in the nuclear regions of Seyfert galaxies (e.g. Tacconi et al. 1996; Helfer \& Blitz 1997).

Previously, we (Curran et al. 2000) found, from a survey of 20 Seyfert galaxies $^{1}$, an $\mathrm{HCN} / \mathrm{CO}$ luminosity ratio

\section{Send offprint requests to: S. Curran,} e-mail: sjc@bat.phys.unsw.edu.au

* Based on results collected at the European Southern Observatory, La Silla, Chile and Onsala Space Observatory, Sweden.

1 These were chosen on the grounds that they provide a good sample of Seyferts. As well as this, they constitute the same sample of Heckman et al. (1989), thus enabling us to also test their finding that type 2 Seyferts have higher molecular gas abundances than type 1s (see Curran 2000a). of $\approx 1 / 6$ for all $13 \mathrm{HCN}$ detections, a ratio similar to that of ULIRGs. We were, however, surprised, to find that such a high HCN/CO luminosity ratio holds for both the "distant" sources (of recessional velocity $v \gtrsim 4000 \mathrm{~km} \mathrm{~s}^{-1}$, where the beamwidth exceeds $\approx 10 \mathrm{kpc}$ ) and the "nearby" sources $\left(v \lesssim 4000 \mathrm{~km} \mathrm{~s}^{-1}\right.$, beamwidth $\left.\lesssim 10 \mathrm{kpc}\right)$, since we expect a larger $\mathrm{CO}$ contribution from the galactic disk in the distant sources, which would result in a lower $L_{\mathrm{HCN}} / L_{\mathrm{CO}}$ ratio compared to the near-by galaxies.

Since the results of Curran et al. (2000) were based on single-beam observation, at the centre of the source, these results suggested that either:

1. For the near-by galaxies we have sampled most of the $\mathrm{CO}$ in our single beam observation;

2. The HCN is more extended than the beam in the nearby sources;

3. Or perhaps the most plausible explanation, simply that the mean luminosity ratio differs between the near-by and distant galaxies.

There may also exist different angular CO distributions between the near-by and distant galaxies, since for the latter sources the high $\mathrm{HCN} / \mathrm{CO}$ luminosity ratio may suggest that the $\mathrm{CO}$ is highly centralised (as is believed 
to be the case for the $\mathrm{HCN}$; within $\approx 1 \mathrm{kpc}$, Downes et al. 1992; Nguyen et al. 1992; Tacconi et al. 1996). Interestingly, the mean values of the far infrared luminosities are $L_{\mathrm{FIR}} \approx 30 \times 10^{10} L_{\odot}$ and $L_{\mathrm{FIR}} \approx 2 \times 10^{10} L_{\odot}$ for the full distant and near-by samples respectively (including the galaxies where Curran et al. 2000 did not detect HCN). In the case of ULIRGs, the higher FIR luminosity $\left(L_{\mathrm{FIR}} \sim 10^{12} L_{\odot}\right)$ is an indicator of a high central CO concentration (Bryant 1997), and the high values of $L_{\text {FIR }}$ in our distant sample may also imply a selection effect, in which our distant sources comprise mainly of galaxies suffering from little $\mathrm{CO}$ contamination from the galactic disk. It is thus probable that in the distant galaxies we sample the whole $\mathrm{CO}$ and HCN. In the near-by sources, however, it is possible that the full distributions are not mapped. In order to account for the limitations of the sampling by our single beam of regions of different linear extent, in Paper I (Curran et al. 2001b) we mapped the distribution of $\mathrm{CO}$ and $\mathrm{HCN}$ in order to assess the contribution of the galactic disk and to take into account the total molecular gas content of each galaxy.

In this paper we shall use the results presented in Paper I to study the $\mathrm{CO}$ to $\mathrm{HCN}^{2}$ luminosity ratios and compare these with the FIR luminosities: In ULIRGs, the relatively high HCN luminosities are believed to be due to the presence of dense star forming cores (Solomon et al. 1992). However, in the case of Seyferts this excess of (denser) gas traced by the HCN (as well as the excess FIR) may also be due to the accumulation of gas around the active nucleus, terminating in the obscuration (Kohno et al. 1999; Curran et al. 2000), and a large fraction of the gas traced by the CO may act as a resevoir for star formation in Seyferts (Curran 2000a; Curran et al. 2001a). In this paper we shall also discuss the $\mathrm{CO} 2 \rightarrow 1 / \mathrm{CO} 1 \rightarrow 0$ ratios for the Southern sources in which we could observe this higher CO transition with SEST.

\section{Results}

\subsection{HCN and CO $1 \rightarrow 0$ distribution in the near-by sample}

In this section we refer to the spectral maps shown in Figs. 1 to 6 of Paper I, which we show, as well as the CO $2 \rightarrow 1$ maps, here as contour plots (Figs. 1 to 17 ). We also describe the gas distribution in individual sources and then we summarise the gas distribution trends.

\subsubsection{NGC 1068}

Although fairly confined $\left(\Theta_{\mathrm{CO}}{ }_{1 \rightarrow 0} \approx \mathrm{HPBW}\right)$, there is still significant amounts of $\mathrm{CO}$ emission at map positions adjacent to the central position, although most of the CO emission is within one $44^{\prime \prime}$ beam from the centre $(\lesssim \pm 3 \mathrm{kpc})$. The HCN shows a similar distribution

\footnotetext{
${ }^{2}$ From now on, unless otherwise stated, HCN or CO refers to the $J=1 \rightarrow 0$ transition for the appropriate molecule.
}

although the emission in the adjacent positions is more marginal.

Even though it is faint (Paper I), CO is still detected out to $\approx 15 \mathrm{kpc}$ from the nucleus thus extending much farther than the HCN (Fig. 21).

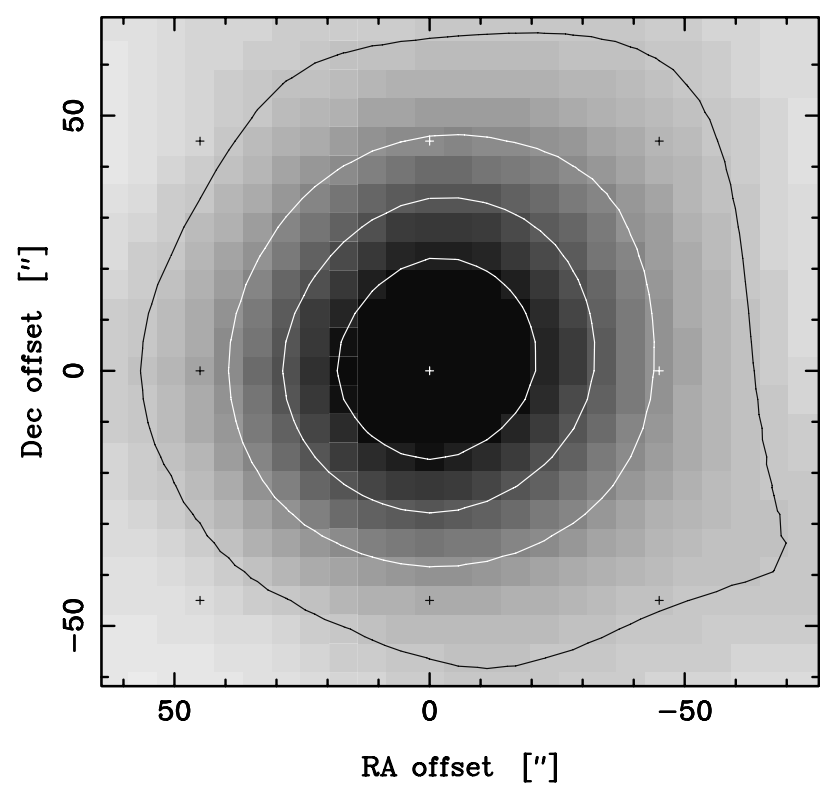

Fig. 1. Contour map of CO $1 \rightarrow 0$ in NGC 1068. The levels are up to $75 \mathrm{~K} \mathrm{~km} \mathrm{~s}^{-1}$ in steps of $15 \mathrm{~K} \mathrm{~km} \mathrm{~s}^{-1}$. Here and in all the contour maps the integrated intensities are uncorrected for beam efficiencies and the observed grid points are shown.

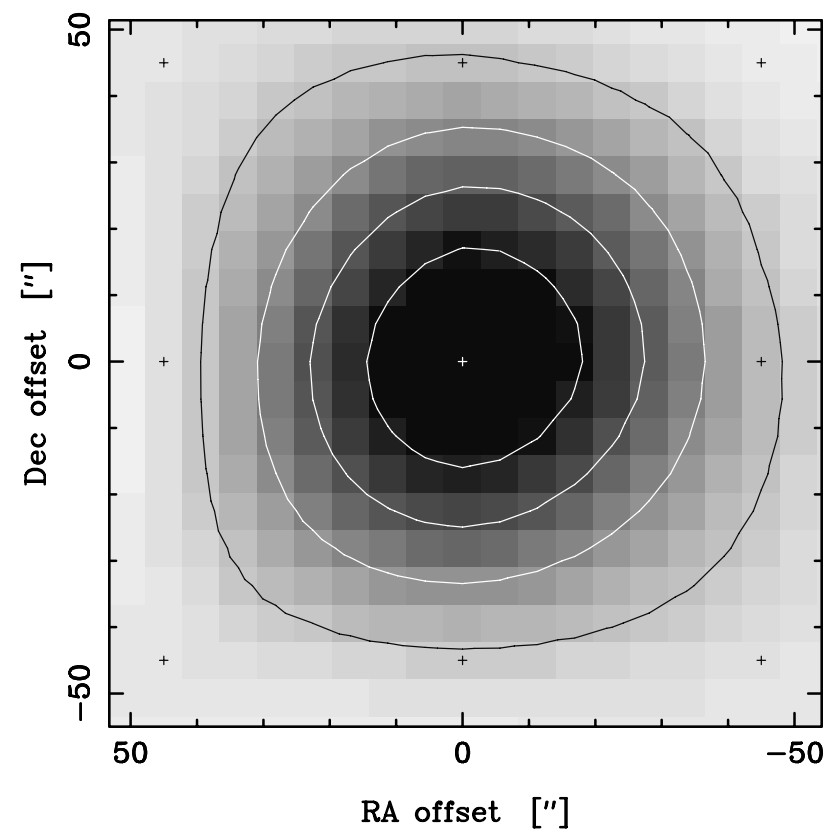

Fig. 2. Contour map of CO $2 \rightarrow 1$ in NGC 1068. The levels are up to $66 \mathrm{~K} \mathrm{~km} \mathrm{~s}^{-1}$ in steps of $\approx 13 \mathrm{~K} \mathrm{~km} \mathrm{~s}^{-1}$. 


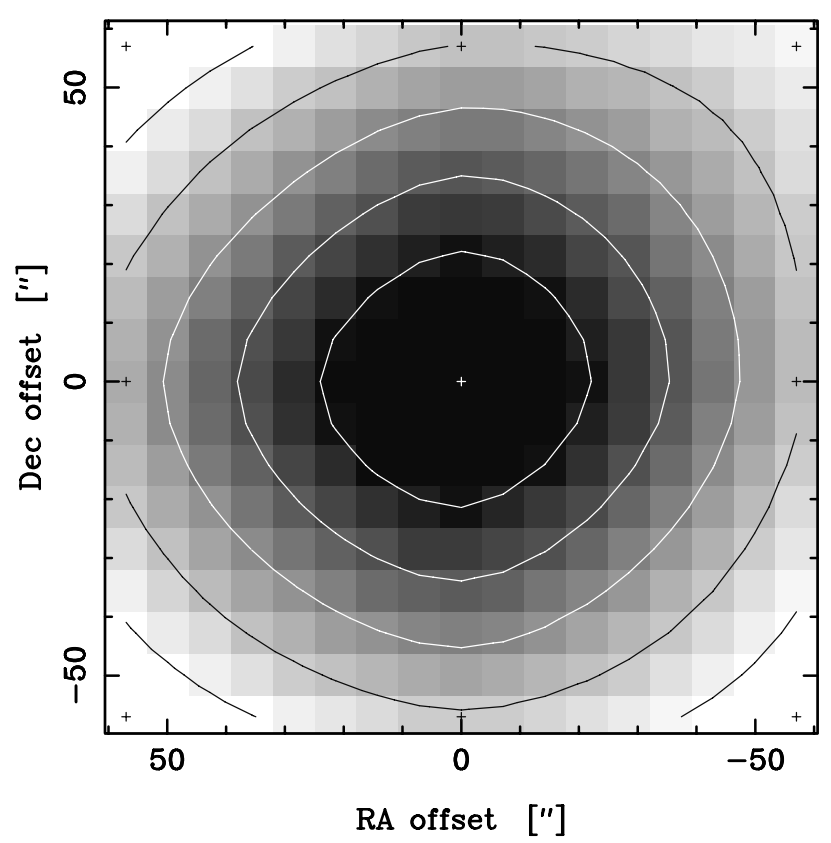

Fig. 3. Contour map of HCN $1 \rightarrow 0$ in NGC 1068. The levels are up to $7 \mathrm{~K} \mathrm{~km} \mathrm{~s}^{-1}$ in steps of $\approx 1.2 \mathrm{~K} \mathrm{~km} \mathrm{~s}^{-1}$.

\subsubsection{NGC 1365}

Like NGC 1068 , we find $\approx 80 \%-90 \%$ of the CO emission to be confined within one $34^{\prime \prime}$ beam from the centre, i.e. $\approx \pm 3 \mathrm{kpc}$ (Sandqvist et al. 1995), probably in the molecular ring (Sandqvist 1999). The HCN results are very interesting in that, despite the fact that this molecule is expected to be confined to within $\approx 1 \mathrm{kpc}$ (Sect. 1 ), we detected it out to map position $\left(34^{\prime \prime}, 68^{\prime \prime}\right)$, i.e. at $\approx 5 \mathrm{kpc}$,

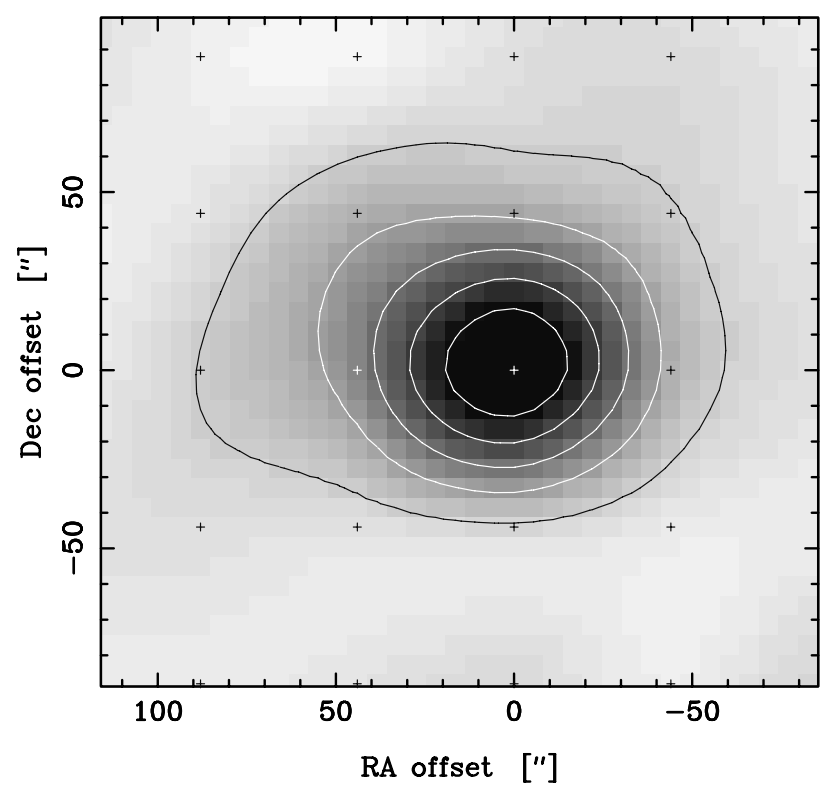

Fig. 4. Contour map of CO $1 \rightarrow 0$ in NGC 1365. The levels are up to $66 \mathrm{~K} \mathrm{~km} \mathrm{~s}^{-1}$ in steps of $11 \mathrm{~K} \mathrm{~km} \mathrm{~s}^{-1}$.

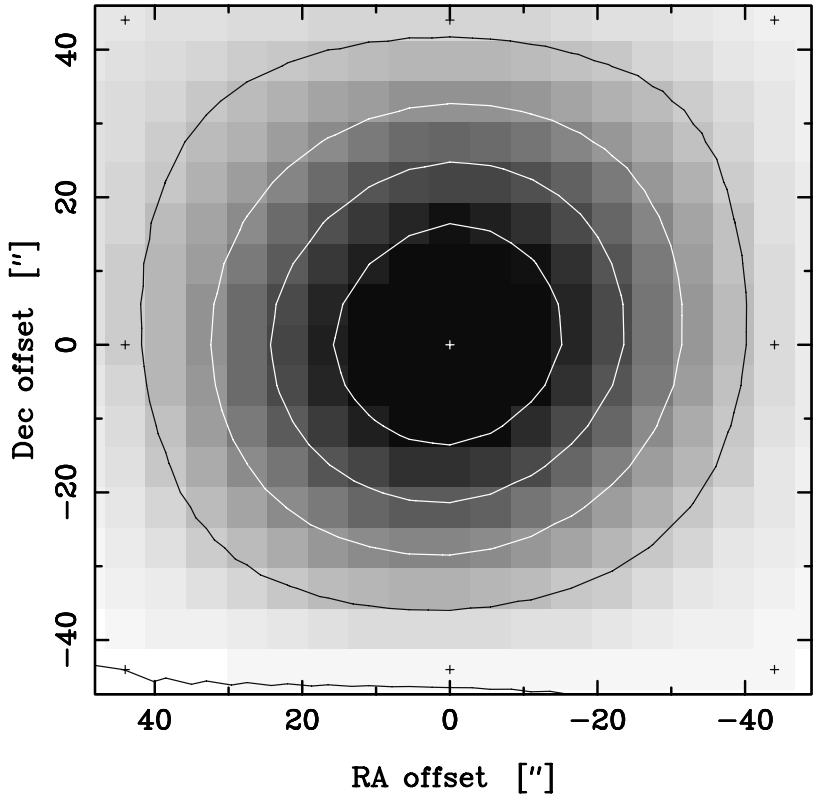

Fig. 5. Contour map of CO $2 \rightarrow 1$ in NGC 1365. The levels are up to $72 \mathrm{~K} \mathrm{~km} \mathrm{~s}^{-1}$ in steps of $\approx 14 \mathrm{~K} \mathrm{~km} \mathrm{~s}^{-1}$.

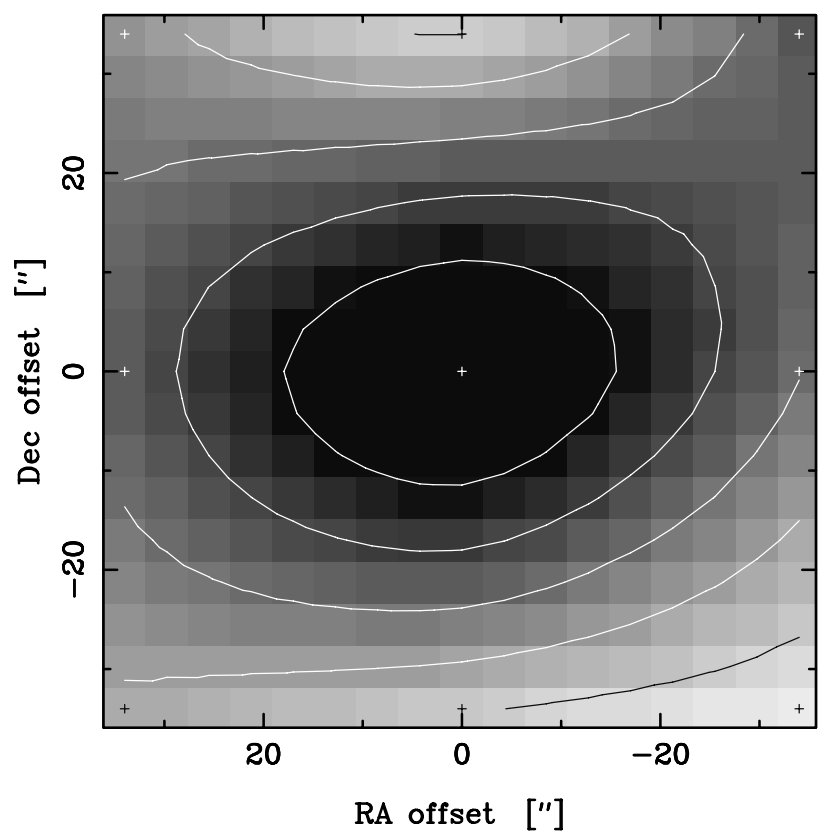

Fig. 6. Contour map of HCN $1 \rightarrow 0$ in NGC 1365. The levels are up to $4 \mathrm{~K} \mathrm{~km} \mathrm{~s}^{-1}$ in steps of $\approx 0.7 \mathrm{~K} \mathrm{~km} \mathrm{~s}^{-1}$.

Fig. $7^{3}$, and obtain a source size of $\approx \pm 2 \mathrm{kpc}$ (Table 1 ). This map position is actually located very close to the major axis of the radio and molecular rings, i.e. at $30^{\circ}$ (Sandqvist et al. 1995; Sandqvist 1999). It should be noted that no detection was made towards the corresponding position in the $\mathrm{SW}\left(210^{\circ}\right)$, and the $\mathrm{CO}$ and radio

\footnotetext{
${ }^{3}$ This actually corresponds to $7 \mathrm{kpc}$ from the central position but in order to account for beam smearing, we have deconvolved (as explained in Paper I) the HCN beam, giving $5 \mathrm{kpc}$.
} 


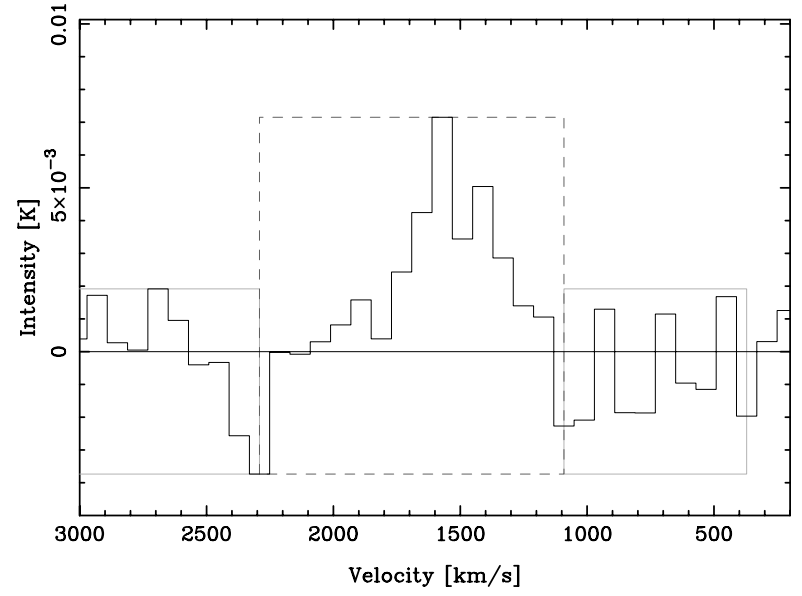

Fig. 7. HCN $1 \rightarrow 0$ at $\left(34^{\prime \prime}, 68^{\prime \prime}\right)$ in NGC 1365 . The velocity resolution is $80 \mathrm{~km} \mathrm{~s}^{-1}$ and the moment and baseline boxes used to determine the main-beam integrated intensity of $2.7 \pm$ $0.7 \mathrm{~K} \mathrm{~km} \mathrm{~s}^{-1}$ are shown. Note that no other HCN was detected at these distances (we mapped to $128^{\prime \prime} \times 128^{\prime \prime}$ ).

emission does appear to be stronger towards the NE (Sandquvist et al. 1995, Paper I)

Our result implies that there is a significant disk contribution to both the $\mathrm{CO}$ and the $\mathrm{HCN}$ luminosity, but the $\mathrm{CO}$ disk is much more extended.

\subsubsection{NGC 2273}

We find the CO emission to be fairly confined; $\approx 60 \%$ of the emission lies within the central beam $( \pm 4 \mathrm{kpc})$, which is not surprising as this is the most distant galaxy of the near-by sample.

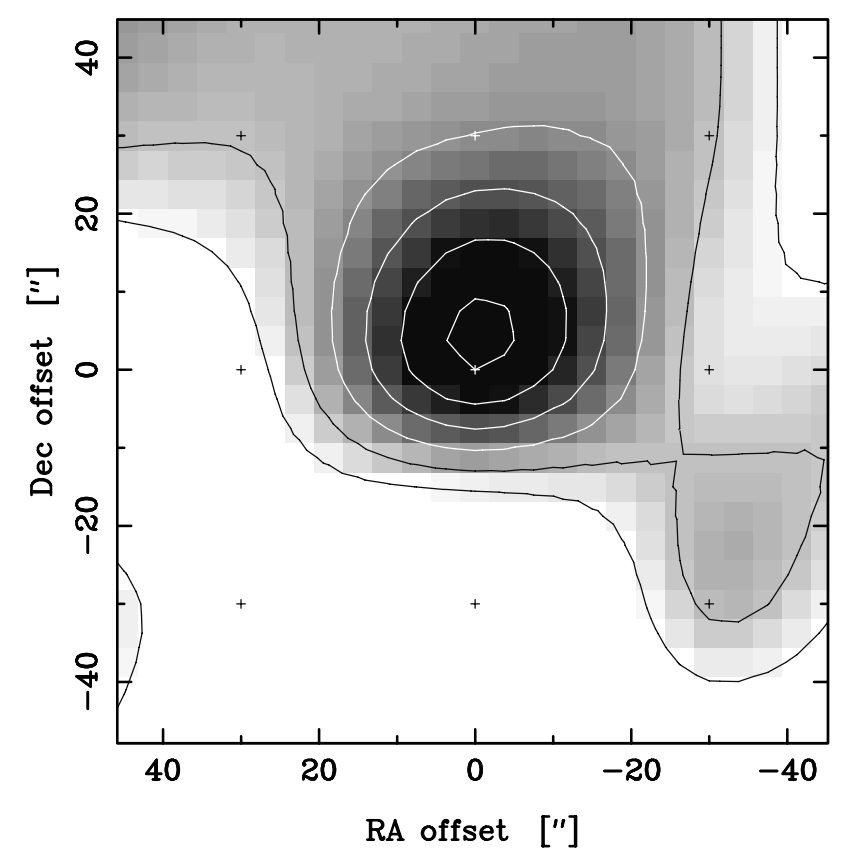

Fig. 8. Contour map of CO $1 \rightarrow 0$ in NGC 2273. The levels are up to $2.3 \mathrm{~K} \mathrm{~km} \mathrm{~s}^{-1}$ in steps of $\approx 0.4 \mathrm{~K} \mathrm{~km} \mathrm{~s}^{-1}$.
Our results imply that there is some disk contribution to the CO luminosity and so we expect the global $\mathrm{HCN}$ to $\mathrm{CO}$ line ratio to be affected by disk contamination.

\subsubsection{NGC 4945}

The CO and HCN may (see Sect. 2.1.8) have similar, and relatively small, source sizes (consistent with the results of Henkel et al. 1994), probably corresponding to the $r \approx 300$ pc ring of Bergman et al. (1992); Dahlem et al. $(1993)^{4}$.

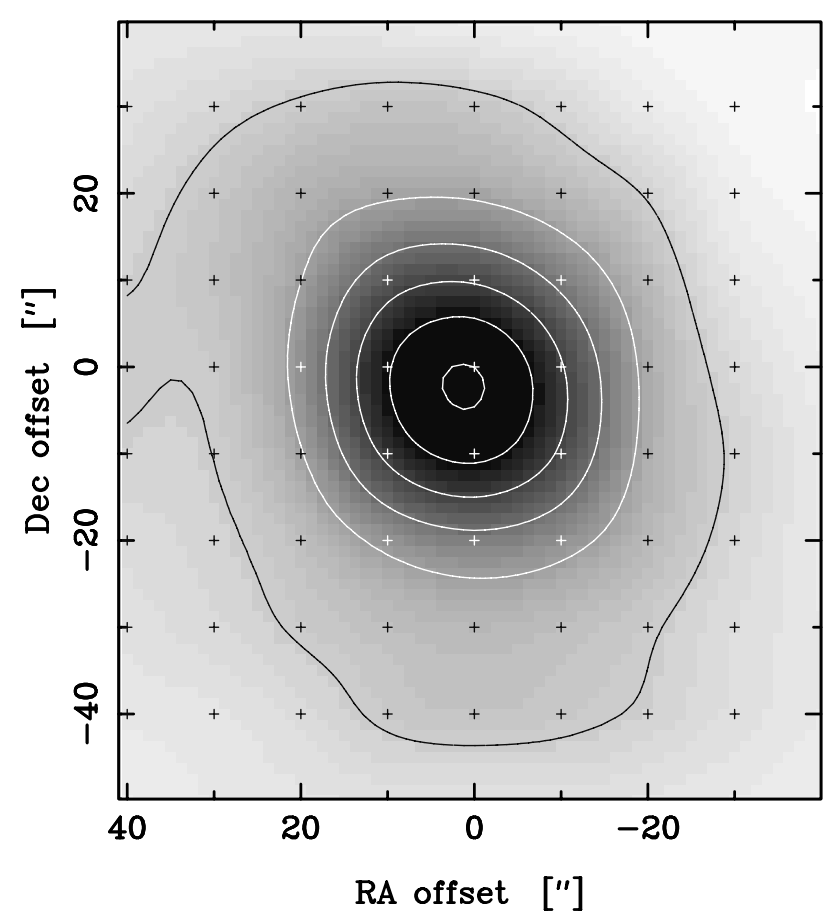

Fig. 9. Contour map of CO $2 \rightarrow 1$ in NGC 4945. The levels are up to $370 \mathrm{~K} \mathrm{~km} \mathrm{~s}^{-1}$ in steps of $\approx 62 \mathrm{~K} \mathrm{~km} \mathrm{~s}^{-1}$. Because of the added position angle to the $\mathrm{CO} 1 \rightarrow 0$ data of Dahlem et al. (1993) it was impossible for us to plot this as a contour plot, which can, however, be seen in their article.

There is a large-scale CO disk in this galaxy (Dahlem et al. 1993) which will also significantly affect the global $\mathrm{CO} / \mathrm{HCN}$ luminosity ratio.

\footnotetext{
${ }^{4}$ Usually, in the absence of maps, the HCN $1 \rightarrow 0$ line is assumed to have the same source size as the $\mathrm{CO} 2 \rightarrow 1$ line, i.e. as Curran et al. (2001a). But here we may find the former to be considerably larger, i.e. $30^{\prime \prime} \mathrm{cf}$. $20^{\prime \prime}$. This would have the effect of increasing the central $\frac{\mathrm{CO} 1 \rightarrow 0}{\mathrm{HCN} 1 \rightarrow 0}$ intensity ratio from $\approx 8$ to $\approx 16$ in Curran et al. (2001a), although it does not significantly change their results as the kinetic temperature and molecular hydrogen density are constrained from the $\mathrm{CO}$ isotopomer intensity ratios. If true, it would, however, lower the density of the dense gas component slightly, which supports the authors' hypothesis that Circinus possesses denser cloud cores in comparison to NGC 4945.
} 


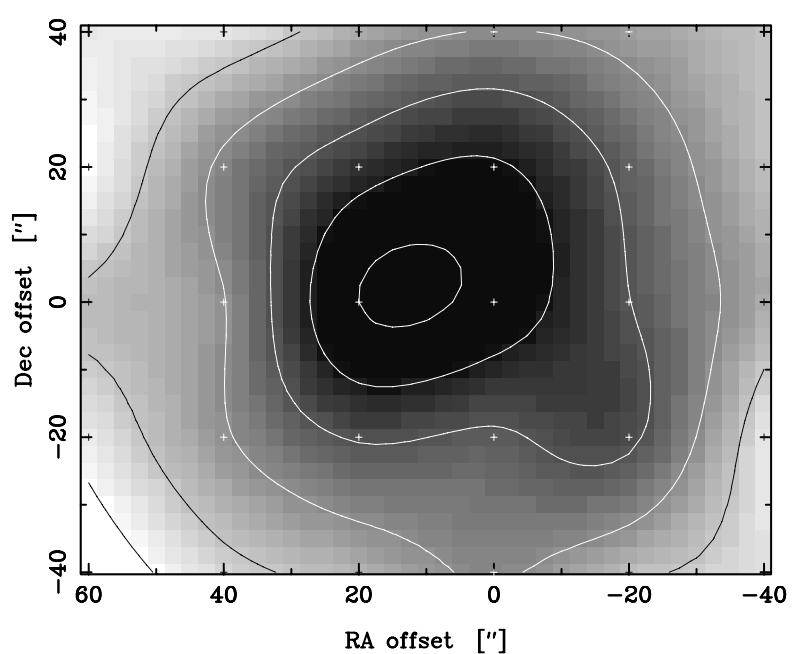

Fig. 10. Contour map of HCN $1 \rightarrow 0$ in NGC 4945. The levels are up to $18 \mathrm{~K} \mathrm{~km} \mathrm{~s}^{-1}$ in steps of $3.6 \mathrm{~K} \mathrm{~km} \mathrm{~s}^{-1}$.

\subsubsection{NGC 5033}

In the nearest galaxy of the original sample (Heckman et al. 1989; Curran et al. 2000), the CO appears to be quite extended and only about $10 \%$ of the emission is in the central beam (Paper I). The optical disk of the galaxy is very extended ${ }^{5}$ and it is possible that the structure seen in our CO map (Fig. 11) is due to spiral arms or other features. Approximately $50 \%$ of the HCN emission is confined within the central beam and its structure is dramatically distinct from any other galaxy in our sample; it appears that the HCN is located in a bar (Fig. 12).

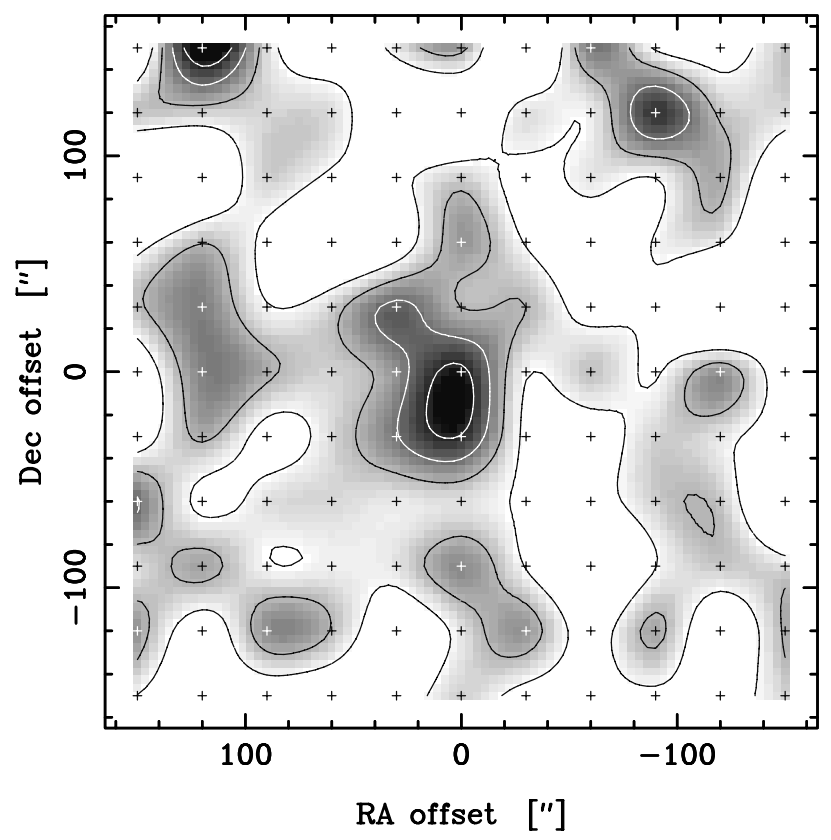

Fig. 11. Contour map of CO $1 \rightarrow 0$ in NGC 5033. The levels are up to $20 \mathrm{~K} \mathrm{~km} \mathrm{~s}^{-1}$ in steps of $5 \mathrm{~K} \mathrm{~km} \mathrm{~s}^{-1}$.

\footnotetext{
${ }^{5}$ Of $\approx 20 \mathrm{kpc}$ radius according to the NASA/IPAC Extragalactic Database.
}

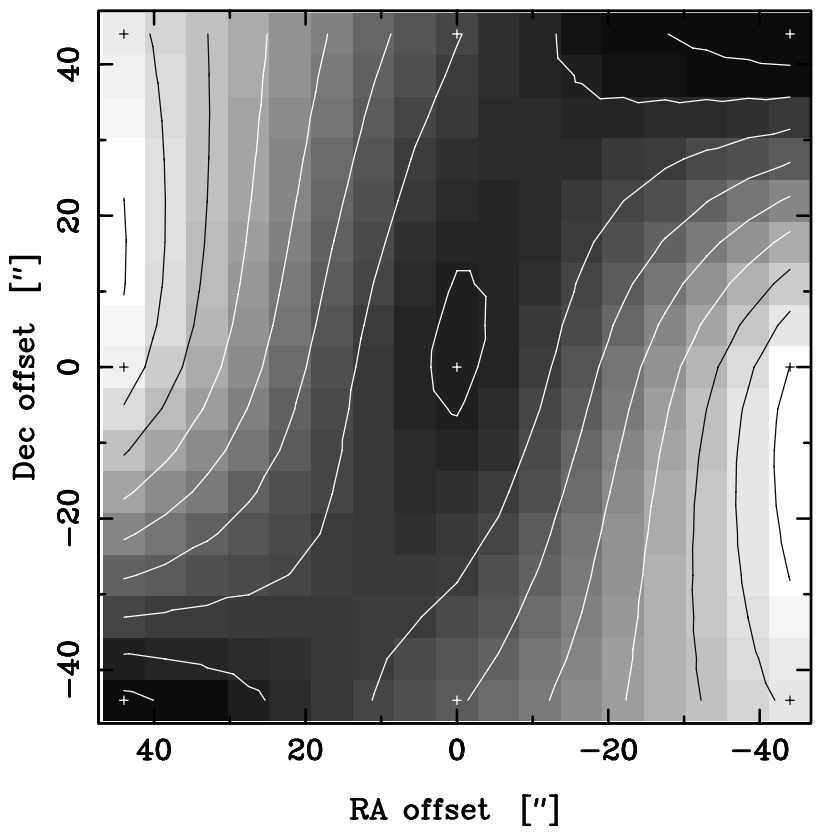

Fig. 12. Contour map of HCN $1 \rightarrow 0$ in NGC 5033. The levels are up to $1.3 \mathrm{~K} \mathrm{~km} \mathrm{~s}^{-1}$ in steps of $\approx 0.1 \mathrm{~K} \mathrm{~km} \mathrm{~s}^{-1}$.

NGC 5033 is a likely example where the disk contribution could dramatically affect the global HCN/CO luminosity ratio.

\subsubsection{Circinus}

As in the case of NGC 4945, the CO and HCN may (see Sect. 2.1.8) share similar source sizes $(r \approx 300 \mathrm{pc}$, corresponding to the molecular ring of Curran et al. 1998) ${ }^{6}$.

The contour maps show that there is significant disk contribution to the CO luminosity which will cause the $\mathrm{HCN}$ to $\mathrm{CO}$ luminosity ratio to decrease with increasing radius.

\subsubsection{NGC 6814}

While there are significant amounts of CO located away from the central beam, most of the emission is confined within the adjacent map positions. Only $\approx 30 \%$ of the $\mathrm{HCN}$ is located within the central beam, and in fact there could be significant emission at $\lesssim 6 \mathrm{kpc}$ (to the east) from the centre. Due to the incomplete map (Paper I) we were unable to produce a contour plot for this molecule.

\footnotetext{
${ }^{6}$ Again this may affect the $\frac{\mathrm{CO} 1 \rightarrow 0}{\mathrm{HCN} 1 \rightarrow 0}$ intensity ratio (Curran et al. 2001a), raising it from $\approx 17$ to $\approx 23$, and, for the same reason as before (Sect. 2.1.4), this does not alter the kinetic temperature and molecular hydrogen density constrained from the $\mathrm{CO}$ isotopomer intensity ratios. The density of the dense gas component could again be lowered but not to the same extent as for NGC 4945.
} 


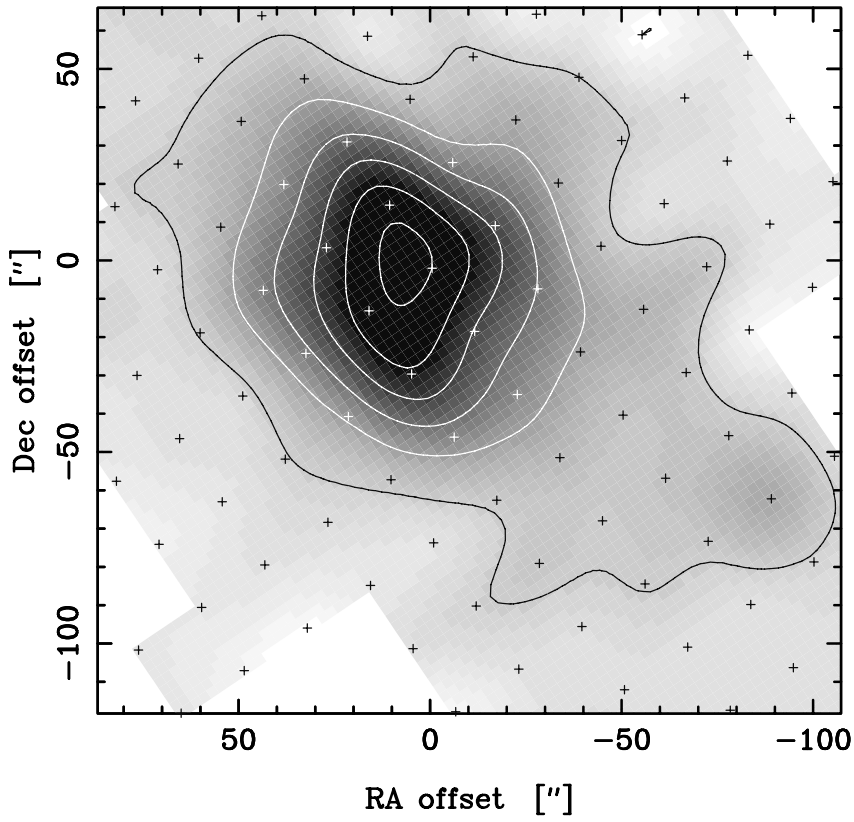

Fig. 13. Contour map of CO $1 \rightarrow 0$ in Circinus. The levels are up to $80 \mathrm{~K} \mathrm{~km} \mathrm{~s}^{-1}$ in steps of $\approx 13 \mathrm{~K} \mathrm{~km} \mathrm{~s}^{-1}$. Note the extension of the emission towards the SW in this and Fig. 14, where this portion of the molecular ring (Curran et al. 1998) is $\approx 1.4$ times more luminous than the NE portion (Curran 1998) corresponding to enhanced star formation (Marconi et al. 1994; Curran 2000b).

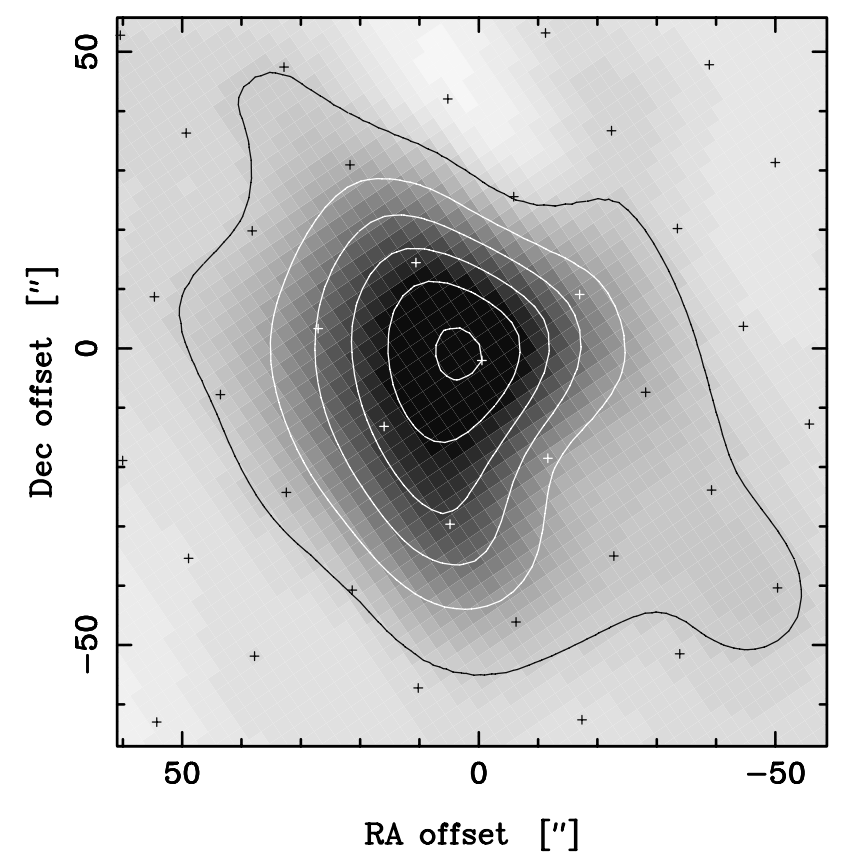

Fig. 14. Contour map of CO $2 \rightarrow 1$ in Circinus. The levels are up to $78 \mathrm{~K} \mathrm{~km} \mathrm{~s}^{-1}$ in steps of $13 \mathrm{~K} \mathrm{~km} \mathrm{~s}^{-1}$.

\subsubsection{Source size fittings}

The source sizes were measured assuming that the source distribution on the sky is Gaussian and deconvolving the telescope response (HPBW) from the full-width halfmaximum (FWHM) diameter (see Paper I). It should be

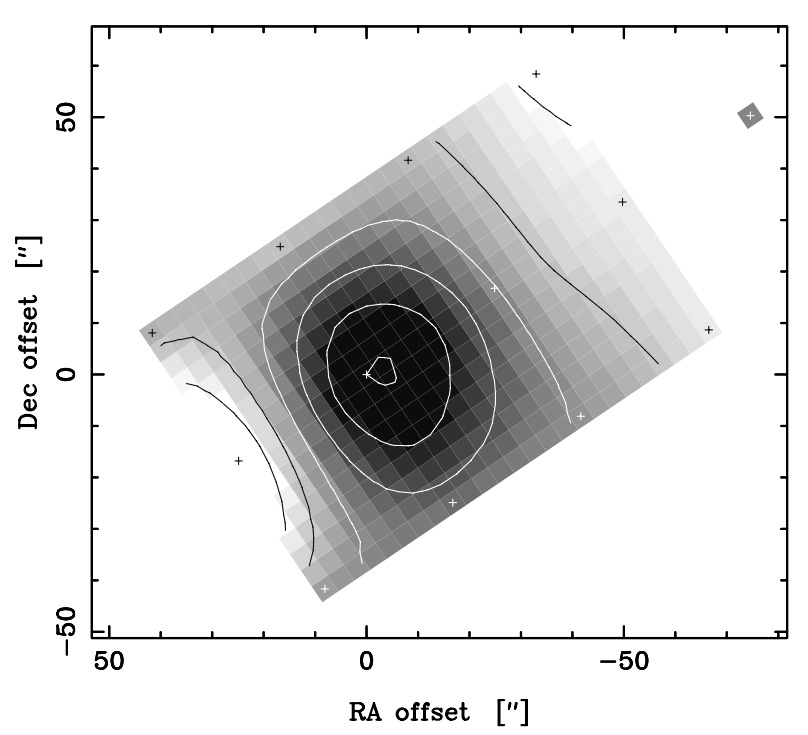

Fig. 15. Contour map of HCN $1 \rightarrow 0$ in Circinus. The levels are up to $5.5 \mathrm{~K} \mathrm{~km} \mathrm{~s}^{-1}$ in steps of $1.1 \mathrm{~K} \mathrm{~km} \mathrm{~s}^{-1}$. Like Figs. 13 and 14 this also shows an extension of emission towards the SW.

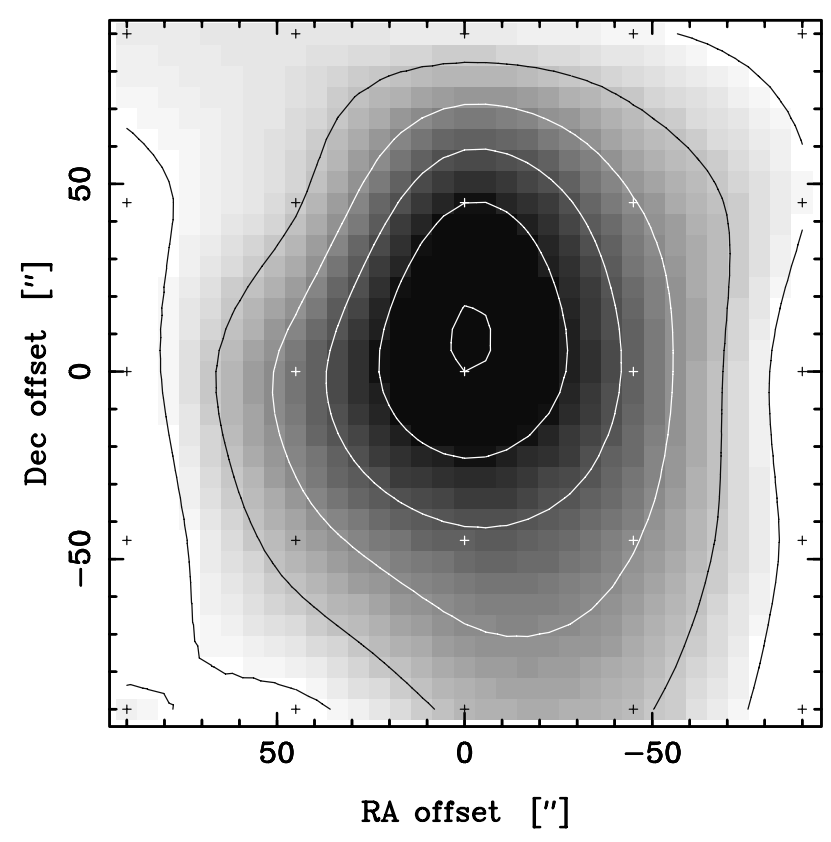

Fig. 16. Contour map of CO $1 \rightarrow 0$ in NGC 6814. The levels are up to $4.7 \mathrm{~K} \mathrm{~km} \mathrm{~s}^{-1}$ in steps of $\approx 0.9 \mathrm{~K} \mathrm{~km} \mathrm{~s}^{-1}$.

noted that the source size concept could be misleading here: The source sizes may seem similar because all galaxies have bright $\mathrm{CO}$ emission coming from their centres, thus causing the source size fits to look the same for $\mathrm{HCN}$ and $\mathrm{CO} 1 \rightarrow 0$. Additionally, for many galaxies (like NGCs 4945 and 5033) there is low level disk emission (which is sub-thermally excited) which will contribute significantly to the luminosity, but only little to the source size fit. We show the fitted source sizes for the different transitions in Table 1. 


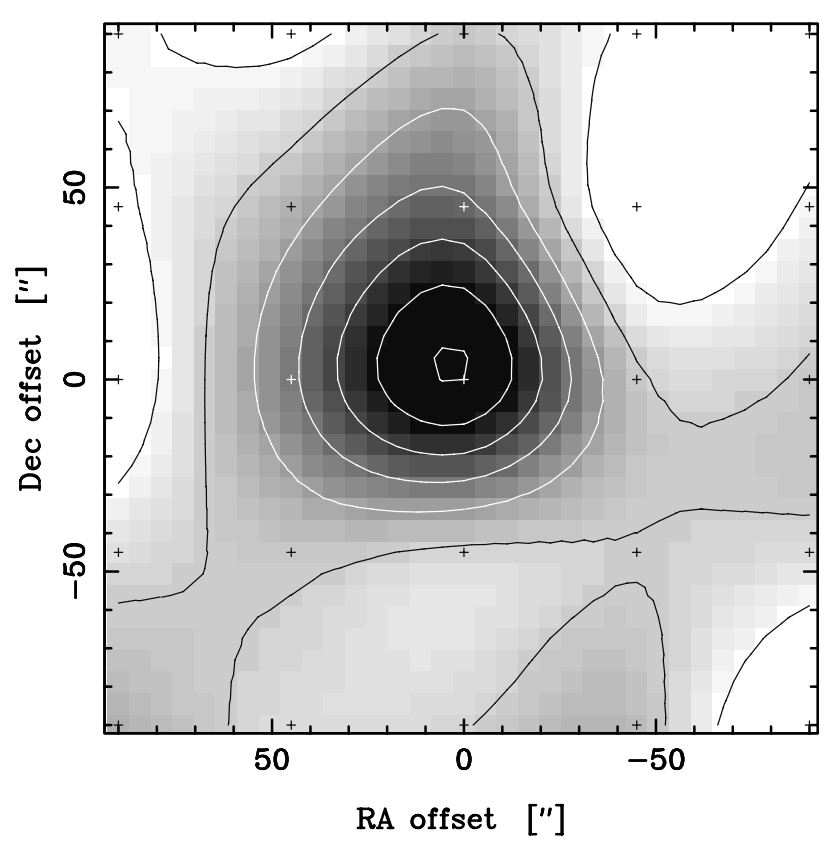

Fig. 17. Contour map of CO $2 \rightarrow 1$ in NGC 6814 . The levels are up to $2.3 \mathrm{~K} \mathrm{~km} \mathrm{~s}^{-1}$ in steps of $\approx 0.4 \mathrm{~K} \mathrm{~km} \mathrm{~s}^{-1}$.

Table 1. Source sizes of the various transitions in the sample expressed as diameters $[\mathrm{kpc}] .{ }^{*}$ All the values for NGCs 2273 (uncertainty $\sim 100 \%$ in CO; HCN was not detected, Paper I), 5033 and 6814 are estimated from the total cf. the average luminosities, as is the HCN value in Circinus (Paper I). Because of the relatively dense map spacing we can include values for CO $2 \rightarrow 1$ in NGC 4945 and Circinus although again these may be misleading (expected to be larger than $\mathrm{HCN} 1 \rightarrow 0$ ) due to the $\mathrm{CO} 2 \rightarrow 1$ beam being that much smaller.

\begin{tabular}{lccc}
\hline Galaxy & $\Theta_{\mathrm{HCN}}$ & $\Theta_{\mathrm{CO} 1 \rightarrow 0}$ & $\Theta_{\mathrm{CO} 2 \rightarrow 1}$ \\
\hline NGC 1068 & $\approx 2-4$ & 3.0 & - \\
NGC 1365 & $\approx 4$ & 4.5 & - \\
NGC 2273 & $?$ & $\sim 7^{*}$ & - \\
NGC 4945 & 0.5 & 0.5 & 0.4 \\
NGC 5033 & $\sim 2^{*}$ & $\sim 2^{*}$ & - \\
Circinus & $\approx 0.8^{*}$ & 0.8 & 0.6 \\
NGC 6814 & $\approx 3.5^{*}$ & $\approx 4^{*}$ & - \\
\hline
\end{tabular}

\subsubsection{Summary}

In general we see that the $\mathrm{HCN}$ is rarely confined to $1 \mathrm{kpc}$ in the FIR weak (near-by) galaxies (Paper I and Table 1) although it does appear to be more concentrated towards the nucleus than the CO (Table 2), thus indicating significant CO contamination in the galactic disk. Returning to Section 1, we can rule out the first option, i.e. that we had previously sampled most of the $\mathrm{CO}$ in the near-by sam$\mathrm{ple}^{7}$, and attribute our previous results to points 2 and 3 (addressed in Sect. 2.2).

7 Although of the near-by galaxies of Curran et al. (2000), NGCs 1068, 1365 and 2273 appear to have their CO fairly confined, as opposed to NGCs 5033 and 6814 (Sects. 2.1.1 to 2.1.7). However, only NGC 2273 has the majority of the CO within the central beam (Table 3 of Paper I).
Table 2. The approximate $\mathrm{CO} / \mathrm{HCN}$ luminosity ratios for the near-by sample over the telescope beam (i.e. as in Curran et al. 2000), over the HCN map and over the full CO mapped region.

\begin{tabular}{lccc}
\hline Galaxy & & $L_{\mathrm{CO}} / L_{\mathrm{HCN}}$ \\
& Beam & HCN Map & CO Map \\
\hline NGC 1068 & 8 & 9 & 20 \\
NGC 1365 & 11 & 11 & 19 \\
NGC 2273 & 5 & - & - \\
NGC 4945 & 13 & 20 & 35 \\
NGC 5033 & 7 & 12 & 27 \\
Circinus & 15 & 33 & 45 \\
NGC 6814 & 9 & 12 & 12 \\
\hline Average & 9 & 16 & 26 \\
\hline
\end{tabular}

\subsection{Luminosity ratios}

In order to simultaneously analyse the two samples, in the following we use the full map (global) values (last column Table 2) for the near-by sample and the central beam values, which are analogous to these, for the distant galaxies.

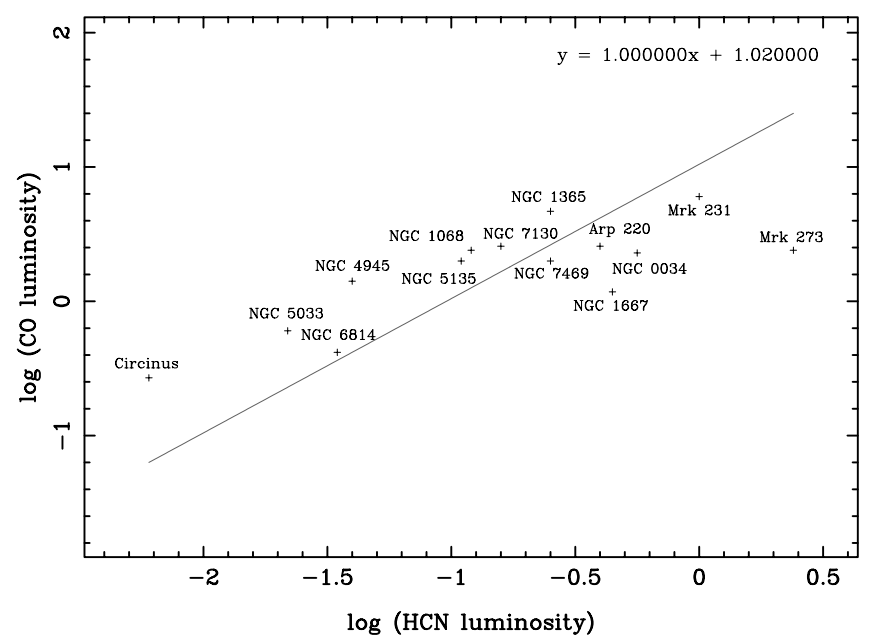

Fig. 18. $\log L_{\mathrm{CO}}$ versus $\log L_{\mathrm{HCN}}$ (units as in Fig. 5 of Curran et al. 2000 i.e. $\times 10^{3} \mathrm{~K} \mathrm{~km} \mathrm{~s}^{-1} \mathrm{kpc}^{2}$ ) for all the detections. From these we obtain a mean fitted ratio of $L_{\mathrm{CO}} / L_{\mathrm{HCN}}=10_{-4}^{+9}$ and using only sources with $v \lesssim 4000 \mathrm{~km} \mathrm{~s}^{-1}$, we obtain a ratio of $L_{\mathrm{CO}} / L_{\mathrm{HCN}}=20_{-10}^{+40}$.

As in Curran et al. (2000), in Figs. 18 and 19 we plot $\log L_{\mathrm{CO}}$ and $\log L_{\mathrm{FIR}}$ against $\log L_{\mathrm{HCN}}$ for all of the sample. Unlike previously, where we obtained $L_{\mathrm{CO}} / L_{\mathrm{HCN}} \approx 6$ for all of the detections and using only sources with $v \gtrsim 4000 \mathrm{~km} \mathrm{~s}^{-1}$, we find a definite difference between the distant and near-by samples, i.e. $L_{\mathrm{CO}} / L_{\mathrm{HCN}}=6 \pm 2$ (distant) and $L_{\mathrm{CO}} / L_{\mathrm{HCN}}=22 \pm 4$ (from Paper I and Table 2). In fact all of the $v \lesssim 4000 \mathrm{~km} \mathrm{~s}^{-1}$ sources lie to the left, above the fitted mean ratio line and, with the exceptions of NGCs $5135\left(v=4112 \mathrm{~km} \mathrm{~s}^{-1}\right)$ and 7130 $\left(v=4842 \mathrm{~km} \mathrm{~s}^{-1}\right)$, the $v \gtrsim 4000 \mathrm{~km} \mathrm{~s}^{-1}$ sources lie to the right, below the line, Fig. 18. 


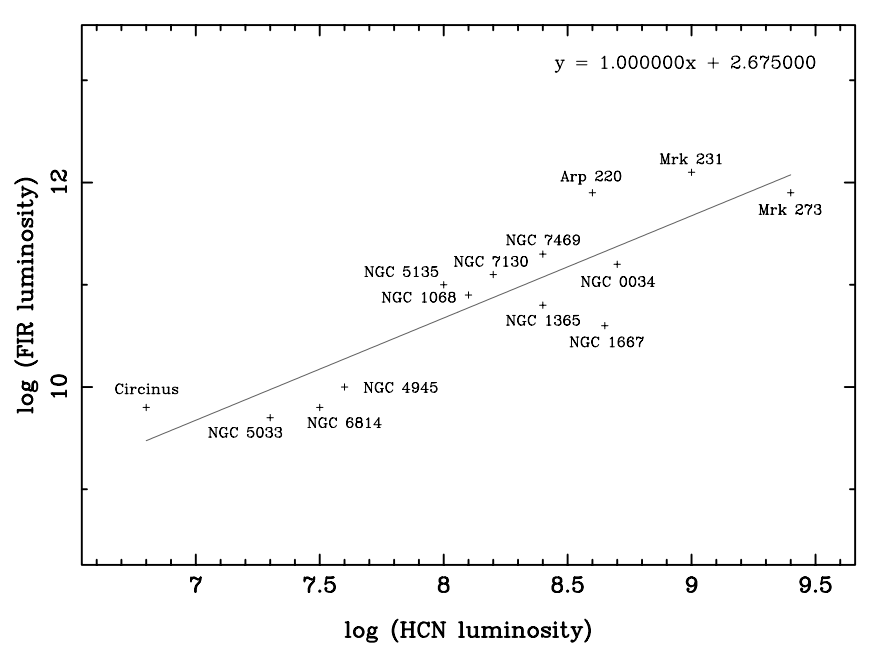

Fig. 19. $\log L_{\mathrm{FIR}}\left[L_{\odot}\right]$ versus $\log L_{\mathrm{HCN}}\left[\mathrm{K} \mathrm{km} \mathrm{s}^{-1} \mathrm{pc}^{2}\right]$ for all the detections.

Again (Curran et al. 2000), in order to determine the far infrared/HCN luminosity correlation, i.e. compare our results with those of Solomon et al. (1992), we plotted $\log L_{\mathrm{FIR}}$ against $\log L_{\mathrm{HCN}}$, for all of the sources, Fig. 19. Once again we see that we obtain a fairly linear (on a log-log plot) relationship between the FIR and the HCN luminosities and from this we determine $L_{\mathrm{FIR}} \approx 500_{-200}^{+400} L_{\mathrm{HCN}} L_{\odot}\left(\mathrm{K} \mathrm{km} \mathrm{s}^{-1} \mathrm{pc}^{2}\right)^{-1}$ (cf. $\approx 600_{-300}^{+600} L_{\mathrm{HCN}} L_{\odot} \quad\left(\mathrm{K} \mathrm{km} \mathrm{s}^{-1} \mathrm{pc}^{2}\right)^{-1}$ previously). For the near-by and distant sources we obtain $L_{\mathrm{FIR}} \approx 350 \quad L_{\mathrm{HCN}} L_{\odot}$ and $L_{\mathrm{FIR}} \approx$ $600 L_{\mathrm{HCN}} L_{\odot}\left(\mathrm{K} \mathrm{km} \mathrm{s}^{-1} \mathrm{pc}^{2}\right)^{-1}$, respectively, suggesting additional FIR flux over and above that associated with the denser molecular gas in the distant sample. All of the near-by sources except NGC 1068 and Circinus lie below the line, i.e. like the distant sample, these two galaxies exhibit an excess in FIR compared to HCN. This also holds when, in order to account for the distances and the extents in our sample (Solomon et al. 1992; Curran et al. 2000), we plot $L_{\mathrm{FIR}} / L_{\mathrm{CO}}$ against $L_{\mathrm{HCN}} / L_{\mathrm{CO}}$, Fig. 20. From this, however, we see a large change in the position of NGC 4945 relative to Circinus in the figure. As suggested by Forbes \& Norris (1998), this may indicate that not all of the FIR in NGC 4945 was sampled by Rice et al. $(1988)^{8}$, although the shift in position for this galaxy does not appear to cause an abnormal deviation from the fit defined by the other Seyferts. We discuss the various luminosity relationships further in Sect. 3.2.

\section{3. $\mathrm{CO} 2 \rightarrow 1 / \mathrm{CO} 1 \rightarrow 0$}

In Table 3 we present the CO $2 \rightarrow 1 / 1 \rightarrow 0$ luminosity ratio for the Southern sources in which we could simultaneously observe the $\mathrm{CO} 2 \rightarrow 1$ and $1 \rightarrow 0$ lines. For the distant sources (NGCs 0034, 4593, 5135, 5548,7130 and 7469 ) we could not map the CO $2 \rightarrow 1$

\footnotetext{
${ }^{8}$ Or that the HCN luminosity of Circinus is relatively weaker, Table 2 .
}

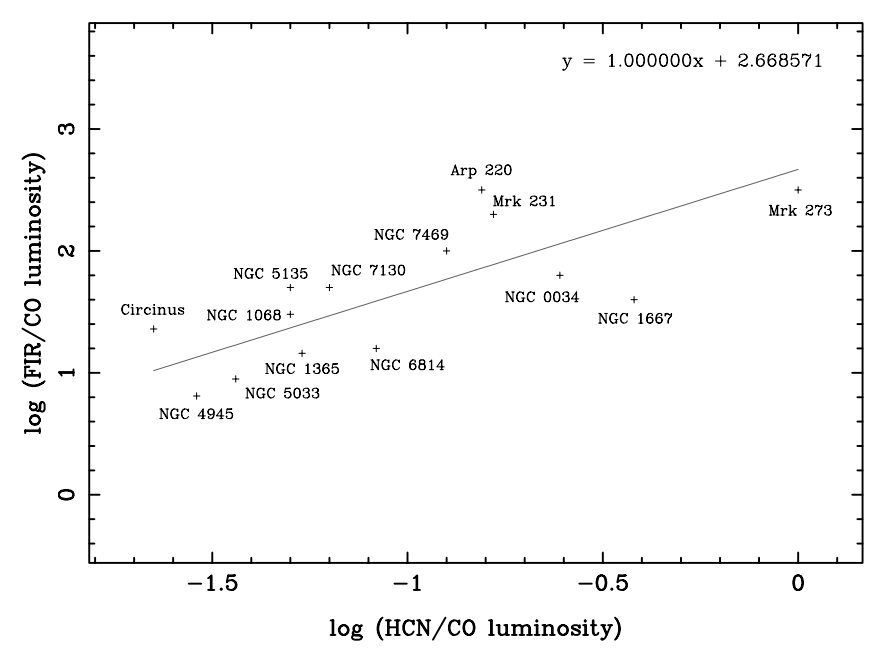

Fig. 20. $\log L_{\mathrm{FIR}} \quad\left[L_{\odot}\left(\mathrm{K} \mathrm{km} \mathrm{s}^{-1} \mathrm{pc}^{2}\right)^{-1}\right] \quad$ versus $\log L_{\mathrm{HCN}}$ normalised by the $\mathrm{CO}$ luminosity for all the detections [no units]. From this fit we also find $L_{\text {FIR }} \approx$ $500 L_{\mathrm{HCN}} L_{\odot}\left(\mathrm{K} \mathrm{km} \mathrm{s}^{-1} \mathrm{pc}^{2}\right)^{-1}$.

Table 3. The global CO $2 \rightarrow 1 / 1 \rightarrow 0$ luminosity/integrated intensity ratios (*and those estimated from the radio continuum) compared with the integrated intensity ratios of Papadopoulos \& Seaquist (1998) (PS98). The final column refers to the radius of the projected $\mathrm{CO} 1 \rightarrow 0$ SEST beam.

\begin{tabular}{lccc}
\hline Galaxy & Ratio & cf. PS98 & $R[\mathrm{kpc}]$ \\
\hline NGC 0034 & $0.45^{*}$ & $0.8 \pm 0.1$ & 8.6 \\
NGC 1068 & $0.7 \pm 0.1$ & $1.1 \pm 0.2$ & 1.6 \\
NGC 1365 & $\approx 1.1 / 0.8^{*}$ & $0.55 \pm 0.09$ & 2.4 \\
NGC 4945 & $\approx 0.4$ & - & 0.8 \\
NGC 4593 & $0.6^{*}$ & - & 3.9 \\
NGC 5135 & $0.7^{*}$ & $0.85 \pm 0.15$ & 6.0 \\
NGC 5548 & $0.73-2.9^{*}$ & - & 7.5 \\
Circinus & $\approx 1.3$ & - & 0.9 \\
NGC 6814 & $0.45 \pm 0.1 / 0.54^{*}$ & $0.6 \pm 0.1$ & 2.3 \\
NGC 7130 & $0.6^{*}$ & $0.5 \pm 0.1$ & 7.0 \\
NGC 7172 & $0.8^{*}$ & - & 3.8 \\
\hline
\end{tabular}

emission, and therefore have no direct information on the $\mathrm{CO}$ source sizes. To estimate the line ratios we have therefore used the suggested correlation between the (low frequency) radio continuum and molecular gas distribution (e.g. Allen 1992; Bajaja et al. 1995). We found radio continuum images for 7 of the sample galaxies and used these to make estimates of the CO source sizes (see Appendix A of Curran et al. 2000b for details).

On the whole, our results seem consistent with those of Papadopoulos \& Seaquist (1998), except in the cases of NGCs 0034 and 1365. In the former galaxy, when we look at the main beam brightness temperatures of Papadopoulos \& Seaquist (1998), we see that they obtain $\approx 10 \mathrm{mK}$ for $\mathrm{CO} 1 \rightarrow 0$ in a $55^{\prime \prime}$ beam and $\approx 7 \mathrm{mK}$ in a 9 point grid map which convolves the $32^{\prime \prime} 2 \rightarrow$ 1 beam to the $1 \rightarrow 0$ beam size. At the distance of NGC 0034 these beam sizes correspond to diameters of $21 \mathrm{kpc}$ and $12 \mathrm{kpc}$ for the $1 \rightarrow 0$ and $2 \rightarrow 1$ transitions, 
respectively. At these linear scales we would be surprised if the molecular gas filled the central beam ${ }^{9}$ in this, one of our distant $\left(v=5931 \mathrm{~km} \mathrm{~s}^{-1}\right)$ sample galaxies. For a point source the intensity ratio should be divided by a factor of $\left(\frac{230 \mathrm{GHz}}{115 \mathrm{GHz}}\right)^{2}=4$ and where the beam is filled no correction is required. So we feel that the Papadopoulos \& Seaquist (1998) ratio would be better quoted as 0.2-0.7 (i.e. somewhere between these two cases). The upper value is still consistent with their stated result and it should be remembered that our ratio is merely an estimate from the radio continuum, although it does lie in the range we obtain for the Papadopoulos \& Seaquist (1998) result and in the two cases where we do have both $\mathrm{CO}$ and radio continuum maps (NGCs 1365 and 6814, Table 3), we find fair agreement in the values. In the case of NGC 1365, we believe that the opposite effect may be the cause of the discrepancy between our ratio and that of Papadopoulos \& Seaquist (1998), i.e. that their $\approx \pm 30^{\prime \prime}$ beam is insufficient to map all of the CO, which is seen out to $\gtrsim \pm 90^{\prime \prime}$ (Fig. 2 of Paper I), in this near-by galaxy. Referring to Table 3 of Paper I, we note a CO $2 \rightarrow 1 / 1 \rightarrow 0$ luminosity ratio of $0.38 \pm 0.08$ over the central beam. This is more consistent with Papadopoulos \& Seaquist (1998) and (unlike NGC 4945 ) the increasing CO $2 \rightarrow 1 / 1 \rightarrow 0$ ratio with galactocentric radius perhaps suggests more vigorous star formation at larger radii (next paragraph).

Note that our two closest examples (which also share similar intrinsic properties, see Curran et al. 2001a), NGC 4945 and Circinus, have the most extreme values (one at either end of the range, Table 3 ) of global $\mathrm{CO} 2 \rightarrow 1 / 1 \rightarrow 0$. The $\frac{\mathrm{CO} 1 \rightarrow 0}{\mathrm{HCN} 1 \rightarrow 0}$ ratio is also significantly higher in Circinus. Comparing the results with those of Curran et al. (2001a) who suggest that star formation may be more dominant in this galaxy, implies that the CO may be excited by large-scale star-burst activity for which the HCN does not necessarily provide a good tracer (Curran et al. 2001a and Sect. 3.2).

\section{Discussion}

\subsection{Gas distributions}

For all of our galaxies the $\mathrm{HCN} / \mathrm{CO}$ luminosity ratio increases towards the nucleus (Table 2), and when we combine our results with those of previous interferometric observations for the much studied NGC 1068,

${ }^{9}$ The maps are not shown in Papadopoulos \& Seaquist (1998). When we compare our values of $T_{\mathrm{mb}}$ with theirs, however, we find that, after correcting for the different NRAO and SEST beams, while the CO $1 \rightarrow 0$ values match between our and their observations, the (total mapped?) $\mathrm{CO} 2 \rightarrow 1$ value of Papadopoulos \& Seaquist (1998) is only about half of ours (Curran et al. 2000). Our only explanation for this is that their $2 \rightarrow 1$ antenna temperature has perhaps not been corrected for main-beam efficiency (our uncorrected antenna temperature better matches their quoted value). In any case it appears as though we have indeed captured all of the CO $2 \rightarrow 1$ flux in the SEST beam.

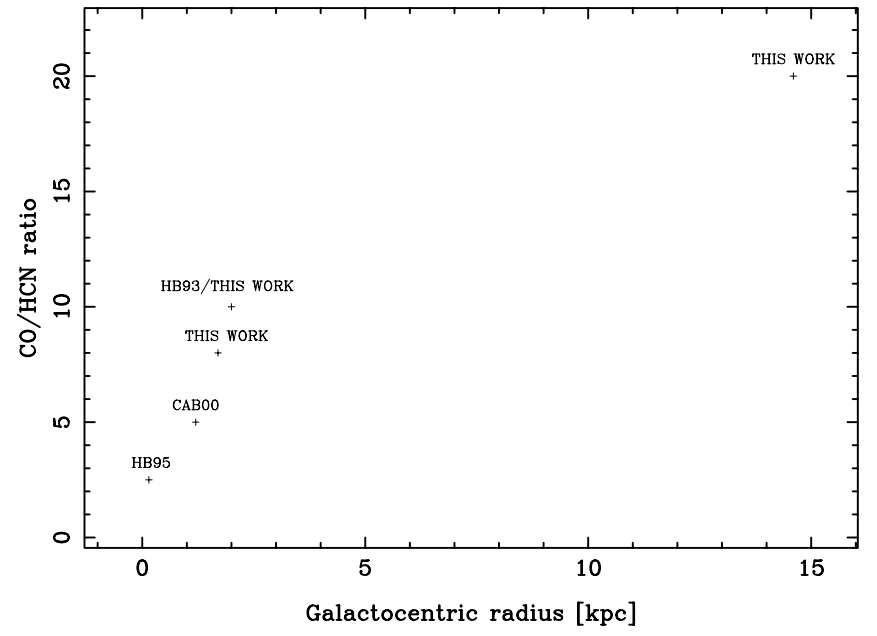

Fig. 21. The CO to HCN intensity/luminosity ratio variation with distance from the nucleus of NGC 1068. HB95 refers to data taken from Helfer \& Blitz (1995), CAB00 from Curran et al. (2000) and HB93 from Helfer \& Blitz (1993). The starburst activity in this galaxy is associated with the $\mathrm{CO}$ ring of radius 0.9-2.4 kpc (Myers \& Scoville 1987; Planeas et al. 1991). Around these radii it is seen that the ratio appears to follow an increasing trend (a detail is shown in Fig. 22) and although there is relatively little $\mathrm{CO}$ out in the galactic disk $(\approx 15 \mathrm{kpc}$, see Paper I), the ratio is very high due to near total lack of $\mathrm{HCN}$.

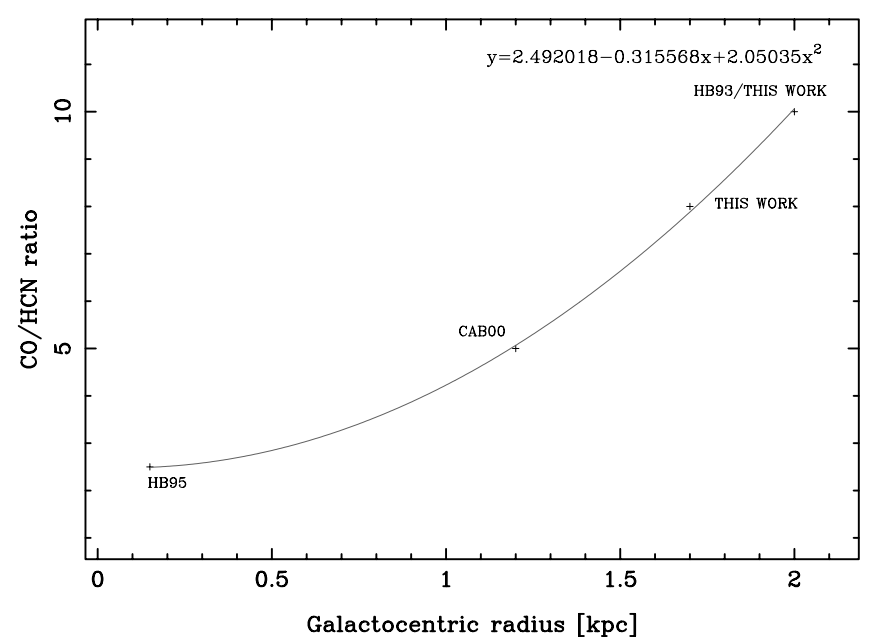

Fig. 22. Detail of Fig. 21 showing the $\mathrm{CO}$ to $\mathrm{HCN}$ intensity/luminosity within the galactic bulge of NGC 1068 (a second order polynomial fit is shown). It is seen here that the relative HCN abundance continues to increase within the inner radius ( $\$ 1 \mathrm{kpc}$ ) of the star forming ring, perhaps supporting the results of Curran et al. (2001a) who suggest that HCN abundances may not be the best tracer of star-burst activity.

Figs. 21 and 22, we find that, while the $\mathrm{HCN} / \mathrm{CO}$ ratio is high within the star-forming region ${ }^{10}$, the ratio keeps increasing within this radius: In AGN the growth of the central super-massive black hole is regulated by the

\footnotetext{
${ }^{10}$ In fact the ratio is comparable to that of the global ratio of the distant sample (Sect. 2.2) and of ULIRGs (Solomon et al. 1992; Helfer \& Blitz 1997).
} 
ongoing star-burst with which it competes for the inflowing material. While the presence of a star-burst will restrict feeding to the black hole, there is expected to be an inner limit to the proximity of this star formation, since tidal effects will exert shear on the parent gas clouds, tearing them apart (e.g. the inner limit for a molecular cloud of density $10^{4} \mathrm{~cm}^{-3}$ approaching a $10^{8} M_{\odot}$ black hole is 55 pc, Lang 1980; Maiolino \& Rieke 1995). As well as this, ionising radiation from the black hole will hinder star formation in molecular clouds (Silk \& Rees 1998). For example, in NGC 7214 the star formation rate is found to increase with distance from the nucleus (Radovich et al. 1998) and in Circinus the star-burst is seen to occur at $\sim 200$ pc from the nucleus (e.g. Marconi et al. 1994) ${ }^{11}$, while in the star-burst galaxy NGC 253, the nucleus is apparently dominated by stars (Ulvestad \& Antonucci 1997; Vogler \& Pietsch 1999). In this, and all galaxies, a supermassive black hole may also exist, although the presence of stars close to this may still be permitted due to the low activity of the black hole (no mega-masers are observed, see Curran 2000b) ${ }^{12}$, which could be the consequence of a low feeding rate (Bryant \& Hunstead 1999). For example, although such a black hole is believed to be present in the Galactic centre (e.g. Kormendy \& Richstone 1995), young stars are observed very close to this (e.g. Okuda et al. 1990; Nagata et al. 1990, 1995).

Finally, from Table 2 and Fig. 21, we see again that contamination by $\mathrm{CO}$ in the disk is indeed an issue, although from the average value of $L_{\mathrm{CO}} / L_{\mathrm{HCN}}$ over the HCN map (Table 2) we can see that any such contamination which may exist in the distant sample is still not sufficient to bring a similar average luminosity ratio between the two samples.

\subsection{Differences between the near-by and distant samples}

Previously, we suggested this difference in the luminosities could be due to a selection effect, i.e. we only detect the FIR bright galaxies at these distances (corresponding to $v \gtrsim 4000 \mathrm{~km} \mathrm{~s}^{-1}$ ) and so the distant sample may be intrinsically different, not only in its FIR and molecular gas luminosities, but in gas distributions to the near-by sample. Now that we have complete CO luminosity values for the near-by sample, we can say that this certainly seems to be a possibility. That is, that in order to produce the high $\mathrm{HCN} / \mathrm{CO}$ ratios in the distant sample we could have relatively little disk contamination, at least in relation to the HCN which seems to be considerably more extended than previously thought ${ }^{13}$ (Sect. 1).

\footnotetext{
11 In fact Maiolino et al. (1998) suggest that the star-burst luminosity on this scale is comparable to the luminosity of the AGN, while being only $\sim 3 \%$ of this within 12 pc of the black hole, thus supporting the notion of an inner limit.

12 http://nedwww.ipac. caltech.edu/level5/Curran/frames.html

13 At least in the near-by sample.
}

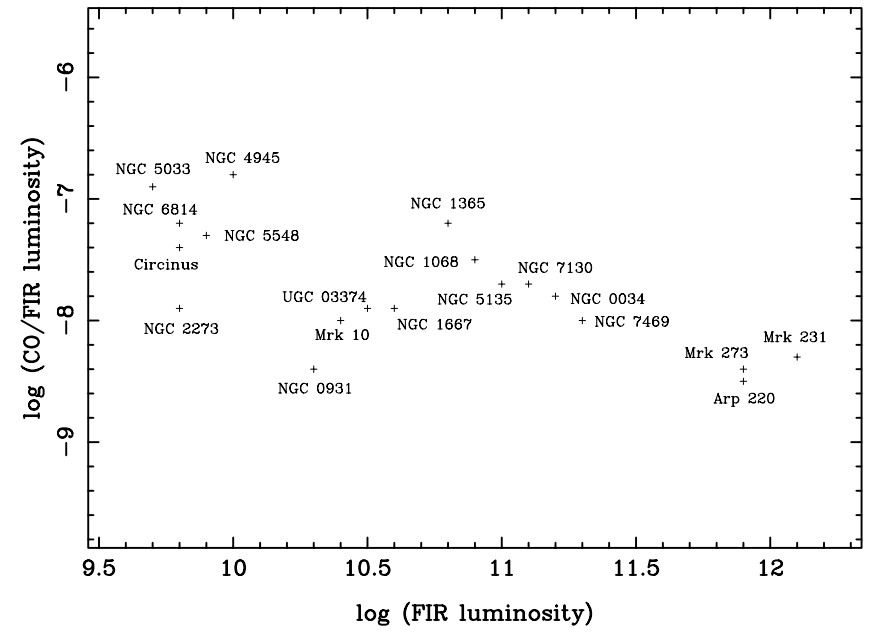

Fig. 23. $\log L_{\mathrm{CO}} / L_{\mathrm{FIR}} \quad\left[\begin{array}{llll}\mathrm{K} \mathrm{km} \mathrm{s}^{-1} & \mathrm{kpc}^{2} & L_{\odot}^{-1}\end{array}\right] \quad$ versus $\log L_{\text {FIR }}\left[L_{\odot}\right]$.

Returning to the ratios, we see a decrease of relative CO luminosity with FIR luminosity (Fig. 23) which implies, not only that the distant sources are more FIR bright (Sect. 1), but that this is in excess relative to CO. This could arise either from young stars and/or the AGN, both of which we would expect to also be traced by the HCN (Curran et al. 2001a).

In the former situation we would expect the $L_{\mathrm{FIR}} / L_{\mathrm{HCN}}$ ratio to be fairly independent of the FIR luminosity of the galaxy and in the latter we might expect this ratio to increase (see Curran et al. 2000), i.e. a further FIR contribution than that of the star formation. Plotting the $L_{\mathrm{HCN}} / L_{\mathrm{FIR}}$ ratio versus $L_{\mathrm{FIR}}$ (Fig. 24) we see that the $\mathrm{HCN} / \mathrm{FIR}$ ratio may well decrease with the FIR luminosity, perhaps indicating that the latter scenario may be the case. Although, as well as the elevated FIR emission due to the accumulation of HCN towards the nucleus (as opposed to star forming regions), the HCN luminosity may also increase accordingly (Curran et al. 2001a and references therein), although Fig. 21 suggests that while the $\mathrm{HCN} / \mathrm{CO}$ ratio is as high as that of ULIRGs over the region of enhanced star formation, there exists excess $\mathrm{HCN}$ at smaller radii, indicating correspondingly higher FIR luminosities at these radii. So rather than a matter of relative luminosities, the question becomes a matter of distribution: How much dense gas/FIR emission arises from the nucleus with respect to the $100 \mathrm{pc}$-scale star forming ring (Curran 2000b and references therein)?

From Figs. 19 and 20 there appears to be no FIR to HCN excess in comparison with normal spirals and ULIRGs (Solomon et al. 1992) ${ }^{14}$. This may be caused by the possibility that both the FIR and HCN emission could

\footnotetext{
${ }^{14}$ From Fig. 2 of Solomon et al. (1992) perhaps it is not so surprising that we find such differences between the near-by and distant samples, as the FIR/HCN relationship spans all the way from normal spirals (e.g. the ratio in Circinus is similar to that of the Milky Way) all the way to ULIRGs, i.e. a range of $L_{\text {FIR }} \sim 10^{10}-10^{12} L_{\odot}$, Figs. 19 and 20 .
} 


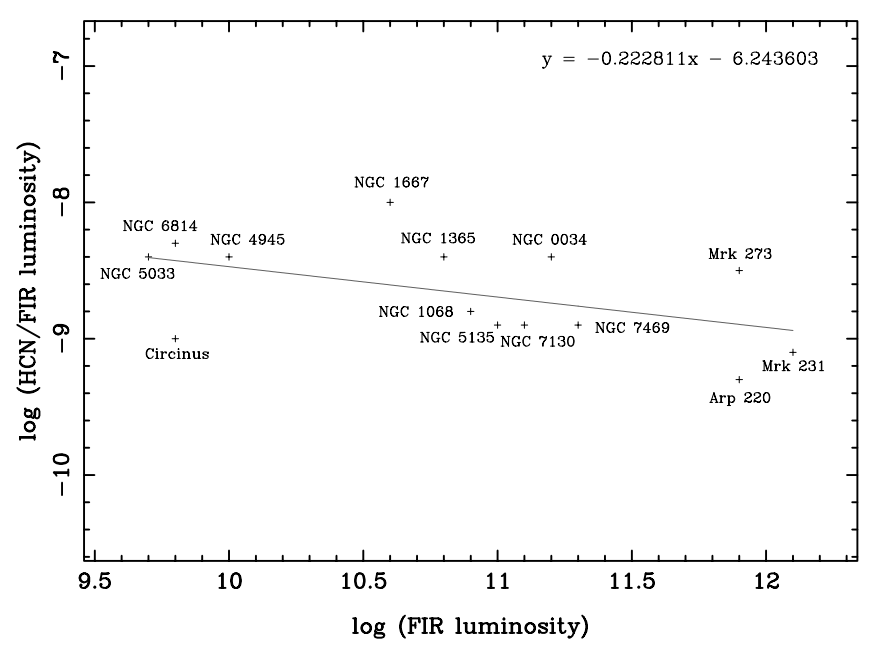

Fig. 24. $\log L_{\mathrm{HCN}} / L_{\mathrm{FIR}}\left[\mathrm{K} \mathrm{km} \mathrm{s}^{-1} \mathrm{kpc}^{2} L_{\odot}^{-1}\right]$ versus $\log L_{\mathrm{FIR}}$ $\left[L_{\odot}\right]$. As in Curran et al. (2000) the least squares linear fit is shown.

come from stars and material surrounding the central engine $^{15}$. In an attempt to distinguish between these two sources of radiation, we note:

1. From above, $\frac{L_{\mathrm{FIR}}}{L_{\mathrm{HCN}}}($ distant $) \approx 2 \frac{L_{\mathrm{FIR}}}{L_{\mathrm{HCN}}}($ near - by $)$, giving a FIR excess in the distant sample;

2. From Fig. 23 we note an excess of FIR in comparison to CO emission in the distant sample. In order to approximately quantify this:

a. From Fig. 18 and Paper I we find $\frac{L_{\mathrm{HCN}}}{L_{\mathrm{CO}}}$ (distant) $\gtrsim$ $2 \frac{L_{\mathrm{HCN}}}{L_{\mathrm{CO}}}($ near - by) i.e. also an excess of $\mathrm{HCN}$ emission in the distant sample.

b. Multiplying this with the previous equation gives $\frac{L_{\text {FIR }}}{L_{\mathrm{CO}}}$ (distant) $\gtrsim 4 \frac{L_{\mathrm{FIR}}}{L_{\mathrm{CO}}}(\text { near }- \text { by })^{16}$.

This is a very rough calculation which assumes an average distant source compared to an average near-by source and the value is probably considerably greater than 4 (i.e. $\approx 10$, Tables 3 and 5 of Paper I). It does show, however, that both HCN and FIR are in excess in the distant sample but that the FIR increases more rapidly than the HCN luminosity with the FIR output of the galaxy. Why should this be if the HCN and FIR are signatures of both star and nuclear activity? One possible answer ${ }^{17}$ could be that while both the line and

$\overline{15}$ Note that in NGC 4194 Aalto \& Hüttemeister (2000) find a deficiency of $\mathrm{HCN}$, although the star forming efficiency may be similar to that of ULIRGs. Also Evans et al. (2001) suggest that the high infrared to CO luminosities in ULIRGs and QSOs may be due to an active nucleus heating the circumnuclear dust.

16 This and the previous point may also indicate a CO excess in the near-by sample which may constitute a resevoir which fuels star formation in the near-by sample. See Curran (2000a) and the following argument.

17 Of course the obvious solution is that, whereas we have sampled most of the HCN (Paper I), in the near-by galaxies, all of the FIR flux may not be sampled. However, comparing the results of the IRAS catalogue (Lonsdale et al. 1985) with those for the large optical galaxies, which are expected to be resolved by IRAS (Rice et al. 1988), we see that for the two galaxies of thermal emission arise from cloud cores and nuclear gas, the beam-filling in the latter would be considerably less than in the more widely distributed HCN associated with star formation (Helfer \& Blitz 1993; Sternberg et al. 1994; Helfer \& Blitz 1995; Tacconi et al. 1998). Since the FIR radiation actually originates from re-radiation by the dust grains (e.g. Rees et al. 1969; Rieke 1978; Lebofsky \& Rieke 1980), we expect this to be a lot more isotropic and therefore not so seriously affected by the beam-filling ${ }^{18}$.

3. Applying this argument to the results of Curran et al. (2001a):

a. Both NGC 4945 and Circinus are well known for their vigorous star-burst activity (e.g. Harnett et al. 1990; Dahlem et al. 1993; Moorwood et al. 1996; Siebenmorgen et al. 1997; Oliva et al. 1999; Viegas et al. 1999), and lie close to the other weaker (near-by) galaxies in Figs. 23 and 24, perhaps suggesting that such Seyfert/star-burst activity may be fairly typical for the near-by sources. However, while in the literature we can find a reference to star-burst activity in each near-by source ${ }^{19}$, we can also find such references for the distant sample ${ }^{20}$;

b. Note that in Fig. 18 Circinus exhibits a definite HCN deficiency (compared to the rest of the sample) and that from Curran et al. (2001a), $\frac{L_{\mathrm{FIR}}}{L_{\mathrm{HCN}}}(\mathrm{NGC} 4945) \approx 0.4 \frac{L_{\mathrm{FIR}}}{L_{\mathrm{HCN}}}$ (Circinus).

our sample in common with the catalogues, NGCs 1365 and 5033 , that all of the FIR seems to have been sampled, even despite the vast extent of NGC 5033 (Paper I and Thean et al. 1997).

18 This could be compounded by the fact that the gas traced by the HCN (and $\mathrm{CO}$ ) is expected to increase in optical depth (e.g. Krolik \& Begelman 1986, 1988) as one approaches the matter responsible for the obscuration of broad lines in (type 2) Seyfert galaxies (e.g. Antonucci 1993; Urry \& Padovani 1995). Therefore there would be line emission only from the facing edge of this in the beam, whereas more of the infrared continuum would be visible through scattering (Miley et al. 1985; Edelson et al. 1987). Note also that by compiling HST (NICMOS) near-infrared $(2 \mu \mathrm{m})$ observations to produce spectral energy distributions, Quillen et al. (2000) find that the mid-infrared opacities in Seyferts could be much less than previously thought. Further to this, due to the large opening angles (see Ulvestad \& Wilson 1984; Wilson \& Tsvetanov 1994; Sandqvist et al. 1995; Curran 2000b, (or http://nedwww.ipac. caltech.edu/level5/Curran/frames.html for a review)) in the obscuring "torus", the radiation from the accretion disk may be able to heat significant portions of the galactic dust located well away from the nucleus.

19 For example, Sandqvist et al. (1988); Arsenault (1989); Baum et al. (1993); Taniguchi \& Ohyama (1998); Chapelon et al. (1999); Grossan et al. (1999); Oliva et al. (1999); Papadopoulos \& Seaquist (1999) and references therein.

${ }^{20}$ For example, Doherty et al. (1995); Calzetti (1997); Richmond et al. (1998); Hill et al. (1999); Anantharamaiah et al. (2000); Carilli \& Taylor (2000); Poggianti \& Wu (2000) and references therein. 
This apparently contradicts point 2 (above) since Curran et al. (2001a) believe that NGC 4945 has greater Seyfert activity and therefore should have more FIR in comparison to HCN emission. The discrepancy is perhaps due to the possibility that not all of the FIR radiation has been sampled in NGC 4945 (Forbes \& Norris 1998) but also, with regard to point 2, that both the galaxies are considerably closer $(\gtrsim 10$ times) than the distant sample. That is, the central HCN does provide ample beam-filling, although this would be confused with $\mathrm{HCN}$ in the star forming rings (Bergman et al. 1992; Dahlem et al. 1993; Marconi et al. 1994; Curran et al. 1998).

Note that for Figs. 23 and 24, the errors (Paper I) as well as variance in the sample could produce an apparent correlation since we are plotting $x / y$ versus $x$. This emphasises the uncertainties in these results making the above points highly speculative. The real solution to the question of relative star-burst/AGN contribution is discussed in Sect. 4.

\subsection{Differences in the dense gas between type 1 and type 2 Seyferts}

Previously (Curran 2000a), we found for the distant sample that both the main Seyfert classes had on average similar abundances of molecular gas, i.e.

$\frac{L_{\mathrm{CO}}}{L_{\mathrm{FIR}}}(\mathrm{Sy} 2) \approx \frac{L_{\mathrm{CO}}}{L_{\mathrm{FIR}}}(\mathrm{Sy} 1)$

$\sim 10^{-8} \mathrm{~K} \mathrm{~km} \mathrm{~s}^{-1} \mathrm{kpc}^{2} L_{\odot}^{-1}$, for all of the sample. However, this result was not so certain with the possibility that

$\frac{L_{\mathrm{CO}}}{L_{\mathrm{FIR}}}(\mathrm{Sy} 2)>\frac{L_{\mathrm{CO}}}{L_{\mathrm{FIR}}}(\mathrm{Sy} 1)$,

which supports the results of Heckman et al. (1989), who find a significant difference in molecular gas abundances between the two main Seyfert classes. However, note that Eq. (1) supports the results of Maiolino et al. (1997) and their sample corresponds to our distant galaxies (i.e. none with $v<4000 \mathrm{~km} \mathrm{~s}^{-1}$ ). When we re-examine our results by adding the global $L_{\mathrm{CO}}$ values for the near-by sample, we find that

$\frac{L_{\mathrm{CO}}}{L_{\mathrm{FIR}}}(\mathrm{Sy} 2) \approx \frac{L_{\mathrm{CO}}}{L_{\mathrm{FIR}}}(\mathrm{Sy} 1)$

$\approx 3 \times 10^{-8} \mathrm{~K} \mathrm{~km} \mathrm{~s}^{-1} \mathrm{kpc}^{2} L_{\odot}^{-1}$, i.e. there is no significant difference in molecular gas abundances between the two main Seyfert classes.

In order to ascertain the implications of this result, i.e. if the fluxes could be of different origins between the two classes, we looked at the HCN/FIR (as well as the $\mathrm{CO} / \mathrm{HCN}$ ) luminosity ratios between the classes. From this it became clear, however, that we would have to consider the distinction between the distant and near-by samples, e.g. obtaining an average of $L_{\mathrm{CO}} / L_{\mathrm{HCN}}$ for one of the Seyfert classes, irrespective of distance, would give a standard deviation which was nearly as large as the average value. Hence further statistics became meaningless, i.e. we have only 3 detections of HCN in Syls (Curran et al. 2000), one of which is in the near-by sample (NGC 6814). This analysis emphasised that although Eq. (3) is valid in that it still holds ${ }^{21}$, there is little point in assigning an average value between the distant and near-by samples, which are evidently quite different. So all we are justified in stating is that

$\frac{L_{\mathrm{CO}}}{L_{\mathrm{FIR}}}(\mathrm{Sy} 2) \approx \frac{L_{\mathrm{CO}}}{L_{\mathrm{FIR}}}(\mathrm{Sy} 1)$

$\approx 1.6 \times 10^{-8} \mathrm{~K} \mathrm{~km} \mathrm{~s}^{-1} \mathrm{kpc}^{2} L_{\odot}^{-1}$ for $v>4000 \mathrm{~km} \mathrm{~s}^{-1}$, and that it is clear that statistics will have to be very much improved in order to determine if the relative $\mathrm{HCN}$ luminosities vary between the two main Seyfert classes. Note that, from the distribution of the number of sources binned according to the flux, Thean et al. (2001) find, on the whole, that Sy2s are marginally more likely to have stronger $60 \mu \mathrm{m}$ infrared emission than Sy1s, perhaps suggesting a more dominant starburst contribution in the former.

\subsection{Sub-thermally excited CO}

Only for four of the galaxies (NGC 1365, NGC 5548, Circinus and NGC 7172) does the CO $2 \rightarrow 1 / 1 \rightarrow 0$ ratio appear to be $\gtrsim 0.8$ (Table 3 ). So for most of the sample the CO emission seems to be sub-thermally excited, indicating densities below $n \lesssim 10^{3} \mathrm{~cm}^{-3}$. This belies the bright HCN emission emerging from several of these objects, e.g. NGC 0034, which suggests gas densities ${ }^{22} n \gtrsim 10^{4} \mathrm{~cm}^{-3}$. This discrepancy could be resolved if the $\mathrm{CO} 1 \rightarrow 0$ source size is larger than that of the $\mathrm{CO} 2 \rightarrow 1^{23}$, so that regions of sub-thermally excited emission lie outside (at larger radii) those of bright $\mathrm{HCN}$ emission, leaving the $\mathrm{CO} 2 \rightarrow 1$ source size closer to that of the $\mathrm{HCN} 1 \rightarrow 0$, although we see from Table 1 that this doesn't appear to be the case. This would also mean that a pointing error would be more serious in the $2 \rightarrow 1$ transition due to the smaller beam and (perhaps) source size. Another possibility is that there is perhaps a mixture of low and high density gas in the inner region, causing a large filling factor of diffuse, unbound low density gas embedding clumps of higher density gas. Models on the production of such a medium have been discussed by Jog \& Das (1992). In order to check this, high resolution, multi-transitional observations of $\mathrm{CO}$ combined with further studies of the emission from high density tracer molecules are necessary,

\footnotetext{
21 Although it is based only on a single Sy1 datum for the near-by sample and two values for the distant sample.

${ }^{22}$ See Curran et al. (2001a) for a detailed analysis of NGC 4945 and Circinus.

${ }^{23} \mathrm{As}$ is found in NGC 4945 (Dahlem et al. 1993; Mauersberger et al. 1996) and the Circinus galaxy (Curran et al. 1998).
} 
although cases of sub-thermally excited CO emission from luminous mergers have been discussed before (e.g. Downes et al. 1992; Aalto et al. 1995). For ULIRGs the situation is even less clear: Many seem to have very sub-thermal global $\mathrm{CO} 2 \rightarrow 1 / 1 \rightarrow 0$ line ratios although we believe that the $\mathrm{CO}$ emission is very concentrated (i.e. no extended disk distribution). For these the idea of a diffuse, low density inter-cloud medium will work very well, thus being both under high pressure and hot, the CO $2 \rightarrow 1 / 1 \rightarrow 0$ line ratio will still be low due to low densities and large velocity gradients.

\section{Conclusions}

From complete mapping of the molecular gas in the nearby sample of Curran et al. (2000) and comparing the results with those for the distant sample, it appears that:

1. We had not previously mapped most of the CO in the near-by galaxies.

2. This is also true for the HCN which is much less confined that previously thought.

3. There does exist a distinct difference between the nearby and distant sample.

Elaborating on this last point, it seems that there is a selection effect in which we only detect the very FIR luminous galaxies $\left(L_{\mathrm{FIR}} \gtrsim 10^{11} L_{\odot}\right)$ at recessional velocities in excess of $\approx 4000 \mathrm{~km} \mathrm{~s}^{-1}$. These galaxies have either incredibly high $\mathrm{HCN} / \mathrm{CO}$ luminosity ratios $(\approx 1$ as in Mrk 273, Curran et al. 2000) within their star forming regions or, unlike the near-by galaxies, the $\mathrm{CO}$ is extremely confined resulting in little contamination by this molecule in the galactic disk.

As well as this we find:

1. Low global CO $2 \rightarrow 1 / 1 \rightarrow 0$ ratios for two thirds of the sample, possibly suggesting sub-thermally excited $\mathrm{CO}$;

2. No significant differences in the CO luminosities between the type 1 and type 2 Seyferts of the sample;

3. That the $\mathrm{HCN} / \mathrm{CO}$ ratio in the near-by sample increases with proximity to the nucleus, possibly further increasing in beyond the region of powerful star formation.

Although this latter point and our other analysis may hint at an increased AGN (cf. stellar) contribution to the FIR emission in the near-by and distant galaxies (respectively), we feel that the key to this puzzle is not improved statistics but actual mapping of the molecular gas distributions in these sources. Such projects would require interferometric observations ${ }^{24}$ which should prove to be worthwhile projects, particularly for the more distant galaxies, when very high resolution interferometers (such as $\mathrm{ALMA}^{25}$ ) become available.

${ }^{24}$ As has already been done for NGC 1068 which appears to confirm our results (Helfer \& Blitz 1995).

${ }^{25}$ For example, using the full $10 \mathrm{~km}$ baseline, a frequency of $\approx 100 \mathrm{GHz}$ will give a linear resolution of $\approx 40 \mathrm{pc}$ at the
Acknowledgements. We wish to thank the referee R. Antonucci for his prompt and helpful comments as well as Kate Brooks at the La Silla $3.6 \mathrm{~m}$ telescope for her advice. This research has made use of the NASA/IPAC Extragalactic Database (NED) which is operated by the Jet Propulsion Laboratory, California Institute of Technology, under contract with the National Aeronautics and Space Administration.

\section{References}

Aalto, S., Booth, R. S., Black, J. H., \& Johansson, L. E. B. 1995, A\&A, 300, 369

Aalto, S., \& Hüttemeister, S. 2000, A\&A, 362, 42

Allen, R. J. 1992, ApJ, 399, 573

Anantharamaiah, K. R., Viallefond, F., Mohan, N. R., Goss, W. M., \& Zhao, J. H. 2000, ApJ, 537, 613

Antonucci, R. R. J. 1993, ARA\&A, 31, 473

Arsenault, R. 1989, A\&A, 217, 66

Bajaja, E., Wielebinski, R., Reuter, H. P., Harnett, J. I., \& Hummel, E. 1995, A\&AS, 114, 147

Baum, S. A., O'Dea, C. P., Dallacassa, D., de Bruyn, A. G., \& Pedlar, A. 1993, ApJ, 419, 553

Bergman, P., Aalto, S., Black, J. H., \& Rydbeck, G. 1992, A\&A, 265, 403

Bryant, J. J., \& Hunstead, R. W. 1999, MNRAS, 308, 431

Bryant, P. M. 1997, Ph.D. Thesis, California Institute of Technology

Calzetti, D. 1997, AJ, 113, 162

Carilli, C. L., \& Taylor, G. B. 2000, ApJ, 532, L95

Chapelon, S., Contini, T., \& Davoust, E. 1999, A\&A, 345, 81

Curran, S. J. 1998, Licentiate Thesis, Chalmers University of Technology

Curran, S. J. 2000a, A\&AS, 144, 271

Curran, S. J. 2000b, Ph.D. Thesis, Chalmers University of Technology

Curran, S. J., Aalto, S., \& Booth, R. S. 2000, A\&AS, 141, 193

Curran, S. J., Johansson, L. E. B., Bergman, P., Heikkilä, A., \& Aalto, S. 2001a, A\&A, 367, 457

Curran, S. J., Johansson, L. E. B., Rydbeck, G., \& Booth, R. S. 1998, A\&A, 338, 863

Curran, S. J., Polatidis, A. G., Aalto, S., \& Booth, R. S. 2001b, A\&A, 368, 824 (Paper I)

Dahlem, M., Golla, G., Whiteoak, J. B., et al. 1993, A\&A, 270, 29

Doherty, R. M., Puxley, P. J., Lumsden, S. L., \& Doyon, R. 1995, MNRAS, 277, 577

Downes, D., Radford, S. J. E., Guilloteau, S., et al. 1992, A\&A, 262,424

Edelson, R. A., Malkan, M. A., \& Rieke, G. H. 1987, ApJ, 321, 233

Evans, A. S., Frayer, D. T., Surace, J., \& Sanders, D. 2001, AJ [astro-ph/0101308]

Forbes, D. A., \& Norris, R. P. 1998, MNRAS, 300, 757

Grossan, B., Spillar, E., Tripp, R., et al. 1999, AJ, 118, 705

Harnett, J. I., Whiteoak, J. B., Reynolds, J. E., Gardner, F. F., \& Tzioumis, A. 1990, MNRAS, 244, 130

Heckman, T. M., Blitz, L., Wilson, A. S., Armus, L., \& Miley, G. K. 1989, ApJ, 342, 735

Helfer, T. T., \& Blitz, L. 1993, ApJ, 419, 86

distance of Mrk 273. Also, being located in Chile, ALMA will prove invaluable in mapping NGC 4945 and Circinus which are too far south to be observed by current arrays. 
Helfer, T. T., \& Blitz, L. 1995, ApJ, 450, 90

Helfer, T. T., \& Blitz, L. 1997, ApJ, 478, 162

Henkel, C., Whiteoak, J. B., \& Mauersberger, R. 1994, A\&A, 284,17

Hill, T. L., Heisler, C. A., Sutherland, R., \& Hunstead, R. W. 1999, AJ, 117, 111

Jog, C. J., \& Das, M. 1992, ApJ, 400, 476

Kohno, K., Kawabe, R., \& Vila-Vilaró, B. 1999, in Proceedings of the 3rd Cologne-Zermatt Symposium, The Physics and Chemistry of the Interstellar Medium, ed. V. Ossenkopf, J. Stutzki, \& G. Winnewisser, 2251

Kormendy, J., \& Richstone, D. 1995, ARA\&A, 33, 581

Krolik, J. H., \& Begelman, M. C. 1986, ApJ, 308, L55

Krolik, J. H., \& Begelman, M. C. 1988, ApJ, 329, 702

Lang, K. R. 1980, Astrophysical Formulae (Berlin: Springer)

Lebofsky, M. J., \& Rieke, G. H. 1980, Nature, 284, 410

Lonsdale, C. J., Helou, G., Good, J. C., \& Rice, W. 1985, Cataloged Galaxies and Quasars Observed in the IRAS Survey (Pasadena: Jet Propulsion Laboratory)

Maiolino, R., Krabbe, A., Thatte, N., \& Genzel, R. 1998, ApJ, 493, 650

Maiolino, R., \& Rieke, G. H. 1995, ApJ, 454, 95

Maiolino, R., Ruiz, M., Rieke, G. H., \& Papadopoulos, P. 1997, ApJ, 485, 552

Marconi, A., Moorwood, A. F. M., Origlia, L., \& Oliva, E. 1994, ESO messenger, 78, 20

Mauersberger, R., Henkel, C., Whiteoak, J. B., Chin, Y. N., \& Tieftrunk, A. R. 1996, A\&A, 309, 705

Miley, G. K., Neugebauer, G., \& Soifer, B. T. 1985, ApJ, 293, L11

Moorwood, A. F. M., Lutz, D., Oliva, E., et al. 1996, A\&A, 315, L109

Myers, S. T., \& Scoville, N. Z. 1987, ApJ, 312, L39

Nagata, T., Woodward, C. E., Shure, M., \& Kobayashi, N. 1995, AJ, 109, 1676

Nagata, T., Woodward, C. E., Shure, M., Pipher, J. L., \& Okuda, H. 1990, ApJ, 351, 83

Nguyen, Q., Jackson, J. M., Henkel, C., Truong, B., \& Mauersberger, R. 1992, ApJ, 399, 521

Okuda, H., Shibai, H., Nakagawa, T., et al. 1990, ApJ, 351, 89

Oliva, E., Origlia, L., Maiolino, R., \& Moorwood, A. F. M. 1999, A\&A, 350, 9
Papadopoulos, P. P., \& Seaquist, E. R. 1998, ApJ, 492, 521

Papadopoulos, P. P., \& Seaquist, E. R. 1999, ApJ, 516, 114

Planeas, P., Scoville, N. Z., \& Myers, S. T. 1991, ApJ, 369, 364

Poggianti, B. M., \& Wu, H. 2000, ApJ, 529, 157

Quillen, A. C., Alonso-Herrero, A., Lee, A., \& Rieke, G. H. 2000, Am. Astron. Soc. Meet., 197, 2104

Radovich, M., Rafanelli, P., \& Barbon, R. 1998, A\&A, 334, 124

Rees, M. J., Silk, J. I., Werner, M. W., \& Wickramasinghe, N. C. 1969 , Nature, 223, 788

Rice, W., Lonsdale, C. J., Soifer, B. T., et al. 1988, ApJS, 68, 91

Richmond, M. W., Filippenko, A. V., \& Galisky, J. 1998, PASP, 110, 553

Rieke, G. H. 1978, ApJ, 226, 550

Sandqvist, A. 1999, A\&A, 343, 367

Sandqvist, A., Elfhag, T., \& Jörsäter, S. 1988, A\&A, 201, 223

Sandqvist, A., Jörsäter, S., \& Lindblad, P. 1995, A\&A, 295, 585

Siebenmorgen, R., Moorwood, A., Freudling, W., \& Käufl, H. A. 1997, A\&A, 325, 450

Silk, J., \& Rees, M. J. 1998, A\&A, 331, L1

Solomon, P. M., Downes, D., \& Radford, S. J. E. 1992, ApJ, 387, L55

Sternberg, A., Genzel, R., \& Tacconi, L. 1994, ApJ, 436, L131

Tacconi, L., Schinnerer, E., Eckart, A., et al. 1998, Astronomische Gesellschaft Meeting Abstracts, Abstracts of Contributed Talks and Posters presented at the Annual Scientific Meeting of the Astronomische Gesellschaft at Heidelberg, September 14-19, 1998, talk \#F01, 14, 01

Tacconi, L. J., Gallimore, J. F., Schinnerer, E., Genzel, R., \& Downes, D. 1996, BAAS, 189, 906

Taniguchi, Y., \& Ohyama, Y. 1998, ApJ, 507, L121

Thean, A., Pedlar, A., Kukula, M. J., Baum, S. A., \& O'Dea, C. P. 2001, MNRAS, in press

Thean, A. H. C., Mundell, C. G., Pedlar, A., \& Nicholson, R. A. 1997, MNRAS, 290, 15

Ulvestad, J. S., \& Antonucci, R. R. J. 1997, ApJ, 488, 621

Ulvestad, J. S., \& Wilson, A. S. 1984, ApJ, 285, 439

Urry, C. M., \& Padovani, P. 1995, PASP, 107, 803

Viegas, S. M., Contini, M., \& Contini, T. 1999, A\&A, 347, 112

Vogler, A., \& Pietsch, W. 1999, A\&A, 342

Wilson, A. S., \& Tsvetanov, Z. I. 1994, AJ, 107, 1227 